(C2019, Elsevier. Licensed under the Creative Commons Attribution-NonCommercialNoDerivatives 4.0 International http://creativecommons.org/about/downloads 


\title{
Flows of real gas in nozzles with unsteady local energy supply
}

\author{
N.A. Brykov , V.N. Emelyanov ${ }^{1}$, A.G. Karpenko², K.N. Volkov ${ }^{3}$ \\ ${ }^{1}$ Baltic State Technical University, 190005, St Petersburg, Russia \\ ${ }^{2}$ St Petersburg State University, 199034, St Petersburg, Russia \\ ${ }^{3}$ Kingston University, SW15 3DW, London, United Kingdom
}

\begin{abstract}
When gas flows at a high speed in a channel with a variable cross sectional area and highintensity energy supply, it experiences complicated physical and chemical processes producing high-temperature gas effects. High-temperature gas effects are a key issue related to design and optimization of nozzles of plasmatron of alternating current. The finite volume method is applied to solve unsteady compressible Euler equations with high-temperature gas effects. Solutions of some benchmark test cases are reported, and comparison between computational results of chemically equilibrium and perfect air flowfields is performed. The results of numerical simulation of one-dimensional and two-dimensional under- and overexpanded nozzle flows with a moving region of energy supply are presented. Output nozzle parameters are calculated as functions of a number and time of burning of plasmatron arcs. The results obtained show a qualitative pattern of gas dynamics and thermal processes in the nozzle with unsteady energy supply demonstrating the displacement of the nozzle shock wave towards the nozzle outlet in the over-expanded nozzle flow in comparison to perfect gas flow.
\end{abstract}

\section{Keywords}

Computational fluid dynamics; Real gas; Nozzle; Plasmatron; Energy supply; Shock wave

\section{Classification codes}

76N15, 76J20, 76N25, 76L05, 76V99

\section{Introduction}

Gas flows in channels with a variable cross-sectional area with unsteady local energy supply arise in alternating current plasmatrons, high-voltage lightning protection switches and other technical devices. In connection with requirements of practice aimed at reducing the number of tests of designed products and the terms of development, there is an increased interest in the mathematical modelling and simulation of flows in nozzles with intensive energy supply [1].

The effect of spatial and temporal characteristics of a pulsating energy source on the supersonic flow in an expanding channel is considered in [2]. The use of low-frequency energy

Corresponding author: k.volkov@kingston.ac.uk 
sources to improve propulsion characteristics produces higher values of specific impulse than a continuous supply of energy.

The transition from a supersonic flow regime in a channel to a subsonic one is accompanied by an increase in static pressure. The interest in the pseudo-combustion mode is due to the fact that during its organization, intensification of the mixing of fuel with an oxidizer occurs, and the rates of chemical reactions increase (due to an increase in pressure and temperature). Controlling the position of the pseudo-shock wave presents a considerable difficulty both in the isothermal flow and in the organization of combustion.

Conventional control methods based on stabilizers (ledges and pylons) lead to total pressure losses, and the position of the pseudo-shock wave is attached to the elements of the stabilizers. The possibilities and methods of controlling a pseudo-shock wave in a smooth channel of constant cross section with a pulse and periodic energy supply are shown in [3]. Design of Laval nozzle for real gas flows is discussed in [4].

The results of calculations of unsteady quasi one-dimensional flow in a channel representing an element of a ramjet engine are reported in [5]. The influence of the parameters of the energy supplied in the pulse and periodic mode (power, pulse frequency, distribution of sources along the channel length) on the flow characteristics is determined.

The behaviour of the electric arc in a supersonic nozzle flow is studied in $[6,7]$. The energy supply leads to the displacement of the nozzle shock towards the inlet section of the channel compared to the flow without an energy supply and to the formation of vortex structures deforming the shape of the nozzle shock. The Prandtl turbulence model and the $k-\varepsilon$ turbulence model are used to close the Reynolds averaged Navier-Stokes (RANS) equations.

A two-dimensional model of flow in a plane and axisymmetric nozzle with local gas heating in the supersonic part, based on the Navier-Stokes equations and equations describing the heat balance in a gas heated by laser radiation, is developed in [8]. Parameters of the gas flow and the volume energy input, at which the gas heating does not exceed 1000-2000 $\mathrm{K}$, are studied. The localized energy input into the flow is accompanied by an increase in pressure in front of the energy release region. When the energy input is sufficiently large, a shock wave occurs, which is located upstream of the energy release region.

A method of numerical simulation of the internal flows of a viscous gas, taking into account non-equilibrium chemical processes and the equilibrium excitation of the internal degrees of freedom of molecules, is developed in [9]. A flow of chemically non-equilibrium gas in a plane channel with a variable cross-sectional area is considered (a five-component model of air is used). The Euler and Navier-Stokes equations for the simulation of the development of zones of local energy supply in supersonic air flow in a channel are used in [10]. In the model of a real gas, the changes in the shock wave structure and flow parameters in the vicinity of the energy supply zone developing in a stationary medium and in the conditions of its interaction with a normal shock at different energy supply intensities, are discussed.

In this study, a model for the numerical study of unsteady gas dynamic effects accompanying local heat release in the subsonic part of a nozzle for a given distribution of power of energy release is developed. The constructed model is applied to flows with unsteady energy supply in nozzles, which are of interest for alternating current plasmatrons. Moving arcs are formed which are sources of intense energy supply. It is allowed to burn one or more arcs that are at different points in space, have different intensities and move at a given speed relative to the flow. A qualitative structure of shock wave and thermal processes in the nozzle during unsteady energy supply is discussed. The dependence of the nozzle output characteristics, as well as the displacement of the nozzle shock wave in over-expanded nozzle flow, on the intensity and cyclical energy supply in the subsonic part of the nozzle is reported. 


\section{Mathematical model}

In simulation of flows with energy supply, the problem is divided into a gas dynamic problem with given heat sources and a physical problem, in which the mechanism of heat release is studied for known flow parameters. The gas dynamic problem is solved using the Euler equations (the viscosity and thermal conductivity of the medium are ignored) or using the Navier-Stokes equations (for a viscous heat conducting gas).

Inviscid flow analysis neglects the effect of viscosity on the flow and is appropriate for high-Reynolds-number applications where inertial forces tend to dominate viscous forces. In particular, inviscid flow calculations are appropriate in an aerodynamic analysis of some high-speed projectile providing a good initial solution for problems involving complicated flow physics and flow geometry [1]. The viscous forces are still important, but in the early stages of calculations the viscous terms in the momentum equations are ignored. Once the calculation has been started and the residuals are decreasing, the viscous terms may be turned on (by enabling a laminar or turbulent flow) and the solution can be continued to convergence.

The unsteady flow of an inviscid compressible gas is described by Euler equations with a source term that takes into account energy supply. To describe flows with gas dynamic discontinuities appearing in high-speed flows, the integral form of the Euler equations is used

$$
\frac{\partial}{\partial t} \int_{V} \boldsymbol{U} d V+\oint_{S} \boldsymbol{F} d S=\int_{V} \boldsymbol{H} d V
$$

The vector of conservative variables, the flux vector and the source term have the form

$$
\boldsymbol{U}=\left(\begin{array}{c}
\rho \\
\rho \boldsymbol{v} \\
e
\end{array}\right), \quad \boldsymbol{F}=\left(\begin{array}{c}
\rho v_{n} \\
\rho v_{n} \boldsymbol{v}+p \boldsymbol{n} \\
(e+p) v_{n}
\end{array}\right), \quad \boldsymbol{H}=\left(\begin{array}{c}
0 \\
0 \\
q
\end{array}\right)
$$

The specific total energy is found as

$$
e=\frac{p}{\gamma-1}+\frac{1}{2} \rho \boldsymbol{v}^{2}
$$

Here, $t$ is the time, $\rho$ is the density, $\boldsymbol{v}$ is the velocity, $p$ is the pressure, $e$ is the total energy per unit mass, $\boldsymbol{n}$ is the external unit normal to the boundary, $v_{n}$ is the normal velocity $\left(v_{n}=\boldsymbol{v} \cdot \boldsymbol{n}=u n_{x}+v n_{y}\right), u$ and $v$ are the Cartesian velocities in $x$ and $y$ coordinate directions, $n_{x}$ and $n_{y}$ are the projections of unit normal on $x$ and $y$ directions, $\gamma$ is the ratio of specific heat capacities, and $q$ is the specific power of energy supply. The intensity of energy supply is specified or found from additional relations taking into account physics of the specific problem. The energy of chemical transformation is the internal energy of the system and is taken into account as an integral part of the total energy. The rate of a chemical reaction is written in the Arrhenius form, and the pre-exponential factor and activation energy are specified for each specific chemical system.

For the model of a stationary one-dimensional flow with energy supply in a narrow zone (reaction front), there is an exact solution linking the flow parameters in the zones before and behind the energy supply. The relationship between the thermodynamic variables before and behind the energy supply zone is described by the Rankin-Hugoniot equation. The pressure and density behind the reaction front are related to the inlet Mach number by the RayleighMichelson equation. The state of the medium behind the energy supply zone is determined from the intersection of the Michelson line with the Rankine-Hugoniot equation. At the same 
time, there is a maximum power supply that can be brought to the flow under steady-state conditions.

The no-penetration boundary condition for normal velocity is applied to the channel wall (the boundary condition for the tangential velocity on the wall is not set in the inviscid gas model). In the inlet section of the channel, the total pressure and total temperature are specified. In the case of a subsonic outflow from the nozzle, a static pressure equal to the surrounding pressure is fixed in the outlet section. This system of conditions determines the physical boundary conditions. Missing boundary conditions are determined from equations written in the characteristic form.

To solve the problem in a stationary formulation, the pseudo-time method is used, according to which the steady state (time independent) distributions of gas parameters are found as a solution of the unsteady problem for $t \rightarrow \infty$. In this case, the form of the initial distribution of the flow quantities is insignificant. To speed up the calculation, physical initial conditions are used, based on the relations of the one-dimensional theory of isentropic gas flow in a nozzle. When solving the problem in an unsteady formulation, the steady state solution of the problem is selected for the initial approximation in the absence of energy supply. The solution of an unsteady problem when applying energy according to a periodic law is carried out until a periodic solution is obtained. The moment of achievement of a periodic solution is determined by comparing average values of the gas flow over a period of time that is a multiple of the period of energy supply (usually after 10 periods).

Negligible viscosity can no longer be assumed near solid boundaries. Assuming inviscid flow can be a useful tool in solving many fluid dynamics problems, however, this assumption requires careful consideration of the fluid sub-layers when solid boundaries are involved [11]. The conventional method for the analysis of supersonic nozzle flowfields is to assume the flow to be inviscid everywhere except near the wall where a thin viscous layer grows. The inviscid core is described by Eulers equations and the wall layer is described by boundary layer equations, or Navier-Stokes equations are applied to flow domain. This technique is universally used to design contoured supersonic nozzles for specified exit flow conditions [12].

However, in supersonic flow, shock and boundary layer interaction is evident, and the structure of this interaction is complex and difficult to predict. Recent investigations in the inviscid and viscous interaction as well as more complex Navier-Stokes codes are encouraging, but still the supersonic flowfields with strong imbedded shock waves and boundary layer separations create tremendous difficulties. In the vicinity of the nozzle exit, the boundary layer at the initial jet expansion is very thin, and inviscid theory has been shown to describe the resulting jet flow reasonably well. Further from the nozzle exit, the thickening shear layer and recirculation region have required the addition of boundary layer approximations to inviscid theory to capture the jet structure fully. The effectiveness of all of these flow solution approaches are highly dependent on specific models applied and how certain models are coupled to the flow solver.

\section{Numerical method}

Two stages of solving the problem at one time step are distinguished: the energy stage (solving the equation of energy change together with the equations describing the heat generation in the flow due to external energy sources) and the gas dynamic stage (calculation of the density, velocity and pressure fields). 
Using the finite volume method, the equation (1) is written in the form

$$
\boldsymbol{U}_{i}^{n+1}=\boldsymbol{U}_{i}^{n}-\frac{\Delta t}{V_{i}} \sum_{j=1}^{N_{e}} \boldsymbol{F}_{i, j} S_{i, j}+\Delta t \boldsymbol{H}_{i}
$$

Here, $\boldsymbol{U}_{i}$ is the vector of conservative variables, $\boldsymbol{F}_{i, j}$ is the flux vector to the cell $i$ through the edge $j, S_{i, j}$ is the edge length $j$ of the cell $i, V_{i}$ is the face area $j$ of the cell $i$.

The Godunov method based on the solution of the Riemann problem is applied. When applying the finite volume scheme (2), the fluxes are calculated in the direction of the normal to the boundary. The flux on the edge of the control volume is determined as

$$
\boldsymbol{F}_{j+1 / 2}=\frac{1}{2}\left[\boldsymbol{F}\left(\boldsymbol{U}_{L}\right)+\boldsymbol{F}\left(\boldsymbol{U}_{R}\right)\right]-\frac{1}{2}|A|\left(\boldsymbol{U}_{R}-\boldsymbol{U}_{L}\right)
$$

where $|A|=R|\Lambda| L$, and $\Lambda=\operatorname{diag}\left\{v_{n}-a, v_{n}, v_{n}+a\right\}$ is the diagonal matrix of eigenvalues of the matrix $A$. For the ideal gas, the Jacobian is represented as

$$
A=\left(\begin{array}{ccc}
0 & 1 & 0 \\
-(3-\gamma) \frac{u^{2}}{2} & (3-\gamma) u & \gamma-1 \\
(\gamma / 2-1) u^{3}-\frac{u a^{2}}{\gamma-1} & (3 / 2-\gamma) u^{2}+\frac{a^{2}}{\gamma-1} & \gamma u
\end{array}\right)
$$

Matrices of the right and left Jacobian eigenvectors have the form

$$
R=\left(\begin{array}{ccc}
1 & 1 & 1 \\
u-a & u & u+a \\
H-u a & u^{2} / 2 & H+u a
\end{array}\right), \quad L=\frac{1}{2}\left(\begin{array}{ccc}
b_{1}+u / a & -b_{2} u-1 / a & b_{2} \\
2-2 b_{1} & 2 b_{2} u & -2 b_{2} \\
b_{1}-u / a & -b_{2} u+1 / a & b_{2}
\end{array}\right)
$$

Here, $a=(\gamma p / \rho)^{1 / 2}$ is the speed of sound, and $b_{1}=b_{2} u^{2} / 2, b_{2}=(\gamma-1) / a^{2}$.

To increase the order of accuracy of the finite volume scheme (3) when interpolating flow quantities on the edge of the control volume, the principle of minimal derivatives is used. The components of the vector of conservative variables on the faces of the control volume are determined by the Roe method. To avoid computational oscillations near the sound point, entropy correction is used. Time discretization is performed using the three-step Runge-Kutta method.

In the computations of inviscid flows some non-physical solutions such as expansion shocks may occur. The non-physical expansion shocks only occur in those regions of the computational domain where expansions are observed through sonic regions. Sonic expansion corresponds to the regions where the wave speed vanishes. Once the region of sonic expansion is detected, an expansion shock can be avoided by diffusing the expansion shock into the domain of computation within the band epsilon. The diffusion process is accomplished numerically by moving eigenvalues of the Jacobian away from its origin. Various formulations could diffuse the expansion shock.

Most high-order techniques experience a loss of robustness when the solution contains discontinuities or even under-resolved physical features. In order to avoid unrealistic solutions like expansion shocks from appearing as a part of a solution, the entropy condition for the Roe scheme must be satisfied. A variety of entropy fix formulae for the Roe scheme have been addressed in the literature $[13,14]$. The most popular is the correction proposed in $[15]$.

In cases where stationary energy supply is carried out in a supersonic flow (the energy supply intensity does not exceed a critical value), it is possible to design time-marching 
calculation schemes [16] (in a supersonic flow, the equations are hyperbolic in the $x$ direction). To speed up the calculations, a vectorized approach to the fluxes calculation is used [17], which allows avoiding the programming of cyclic constructions.

\section{Real gas effects}

At low temperatures and pressures, internal energy consists of the energy of the translational and rotational motion of molecules. Therefore, air is considered as a perfect diatomic gas with a constant molecular weight, constant specific heats and constant adiabatic index. High-temperature flows are characterized by the excitation of vibrational degrees of freedom of polyatomic molecules, the presence of dissociation and ionization processes [1]. In the air, oxygen dissociation occurs at $T=2000-4000 \mathrm{~K}$, and nitrogen dissociation occurs at $T=4000$ $10000 \mathrm{~K}$. At $T=7000-10000 \mathrm{~K}$, the ionization process begins with the formation of free electrons. At $T>10000-12000 \mathrm{~K}$, significant fractions are single ions of these components.

To describe the equilibrium dissociation of some diatomic gases, the model of the ideal dissociating gas (Lighthill model) is used. Its properties are described by three constants, which allows for ja generalized analysis of the influence of dissociation. In the model developed in [18], air is considered as an ideal mixture of oxygen and nitrogen (it is assumed that there are no nitrogen compounds with oxygen) with constant molar concentrations, taking into account the excitation of vibrational and rotational degrees of freedom of molecules. The average molar mass of the mixture remains constant, and the equation of state retains the form corresponding to the equation of state of an ideal gas.

The advantage of the model proposed in [19] (the Kraiko model) is the inclusion of dissociation and ionization of air at high temperatures. When taking into account the real thermodynamic properties of air, explicit expressions are used for the density and specific internal energy through pressure and temperature $\rho=\rho(p, T)$ and $\varepsilon=\varepsilon(p, T)$. In the temperature range from 200 to $20000 \mathrm{~K}$ and pressures from 0.001 to $1000 \mathrm{~atm}$, the model error does not exceed $1.5 \%$ in density and $3 \%$ in enthalpy.

The equations describing the flow of a real gas have the same form as the equations for an ideal gas (1). When using the approximate model (the Kraiko model or the Lighthill model), difficulties arise in the transition from conservative to physical variables. In approximate models, the gas state is determined in the function of the variables $\rho=\rho(p, T)$ and $\varepsilon=$ $\varepsilon(p, T)$, and the dependence $p=p(\rho, \varepsilon)$ is used for calculations. For the transition between physical and conservative variables with known density and internal energy, a system of algebraic equations is solved

$$
f_{1}(p, T)-\rho=0, \quad f_{2}(p, T)-\varepsilon=0 .
$$

In addition to the conservative form of equations, a quasi-linear form of equations is used in the calculations (for example, for specification of boundary conditions, determination of characteristic relations, calculation of eigenvectors). Unlike the perfect gas model, in the real gas model the Jacobian has the form

$$
A=\left(\begin{array}{ccc}
0 & 1 & 0 \\
a^{2}-u^{2}-\left(H-u^{2}\right) p_{\varepsilon} / \rho & 2 u-u p_{\varepsilon} / \rho & p_{\varepsilon} / \rho \\
u\left(a^{2}-H\right)-u\left(H-u^{2}\right) p_{\varepsilon} / \rho & H-u^{2} p_{\varepsilon} / \rho & u+u p_{\varepsilon} / \rho
\end{array}\right)
$$

For a gas whose thermal equation of state is written in the form $p=p(\rho, \varepsilon)$, the matrices 
composed of the right and left Jacobian eigenvectors have the form [20,21]

$$
\begin{gathered}
R=\left(\begin{array}{ccc}
1 & 1 & 1 \\
u-a & u & u+a \\
H-u a & H-\rho a^{2} / p_{\varepsilon} & H+u a
\end{array}\right), \\
L=\frac{p_{\varepsilon}}{2 \rho a^{2}}\left(\begin{array}{ccc}
u^{2}-H+\rho c(u+a) / p_{\varepsilon} & -u-\rho a / p_{\varepsilon} & 1 \\
2\left(H-u^{2}\right) & 2 u & -2 \\
u^{2}-H-\rho c(u-a) / p_{\varepsilon} & -u+\rho a / p_{\varepsilon} & 1
\end{array}\right) .
\end{gathered}
$$

The total energy and local speed of sound are found as

$$
H=\varepsilon+\frac{p}{\rho}+\frac{1}{2} u^{2}, \quad a^{2}=p_{\rho}+p_{\varepsilon} \frac{p}{\rho^{2}},
$$

where $p_{\rho}=\partial p / \partial \rho, p_{\varepsilon}=\partial p / \partial \varepsilon$. The Jacobian eigenvalues and characteristic ratios for real gas are preserved in the same form as in the case of an ideal gas flow. The vectors of conservative variables on the faces of the control volume are determined by the Roe method in the same relationships as for an ideal gas.

Methods for approximating thermodynamic functions are based on the use of an effective adiabatic index. The adiabatic exponent is replaced by a certain constant value, which is formally considered as an adiabatic exponent and corresponds to it with unexcited degrees of freedom of gas molecules. There are various approaches to determining the effective adiabatic exponent. In modelling shock wave processes, the definition of $\gamma_{s}=h / \varepsilon$ is used. Using pressure and density as thermodynamic parameters, the internal energy is represented in the form $\varepsilon=p /\left[\left(\gamma_{s}-1\right) \rho\right]$. The isentropic index (effective specific heat ratio) is determined by the formula

$$
\gamma_{e}=\frac{c_{p}}{c_{v}}\left[1+\frac{p}{\mu}\left(\frac{\partial \mu}{\partial p}\right)_{T}\right]^{-1},
$$

where $\mu$ is the molar mass of gas, $c_{p}$ and $c_{v}$ are specific heat capacities at constant pressure and constant volume. At a pressure-dependent molecular weight, the adiabatic exponent $\gamma_{e}$ does not equal (like $\gamma_{s}$ ) the ratio of specific heat capacities in the corresponding process. The values $\gamma_{c}=c_{p} / c_{v}, \gamma_{s}$ and $\gamma_{e}$ depend on the pressure and temperature as shown in the Figure $1 \mathrm{a}-\mathrm{c}$. The distribution of effective heat ratio as a function of temperature at a fixed pressure equal to $p=1$ atm shows the Figure 1d.

A solution of the Riemann problem for real gas is provided in [22]. The splitting of the flow vector and the increment of the flow vector for the case of a real gas are considered in $[23,24]$, and features of the Roe method are discussed in [20]. The case of a multidimensional flow is considered in [24], while various methods of splitting the flow vector are discussed in [25]. When using the Godunov method, it is assumed that the solution to the Riemann problem corresponds to the case of a frozen flow (for neighbouring cells, their effective values of the adiabatic exponents are used). The resulting frozen flows are used to calculate conservative variables, after which equilibrium parameters are determined for each cell.

Entropy and enthalpy are calculated with the equilibrium adiabatic index $\gamma_{e}$. Moreover, the equilibrium speed of sound is found from the relation $a_{e}=\left(\gamma_{e} p / \rho\right)^{1 / 2}$. The characteristic conditions are set similarly to the case of an ideal gas, taking $a^{2}=p_{\rho}+p p_{\varepsilon} / \rho^{2}$. 

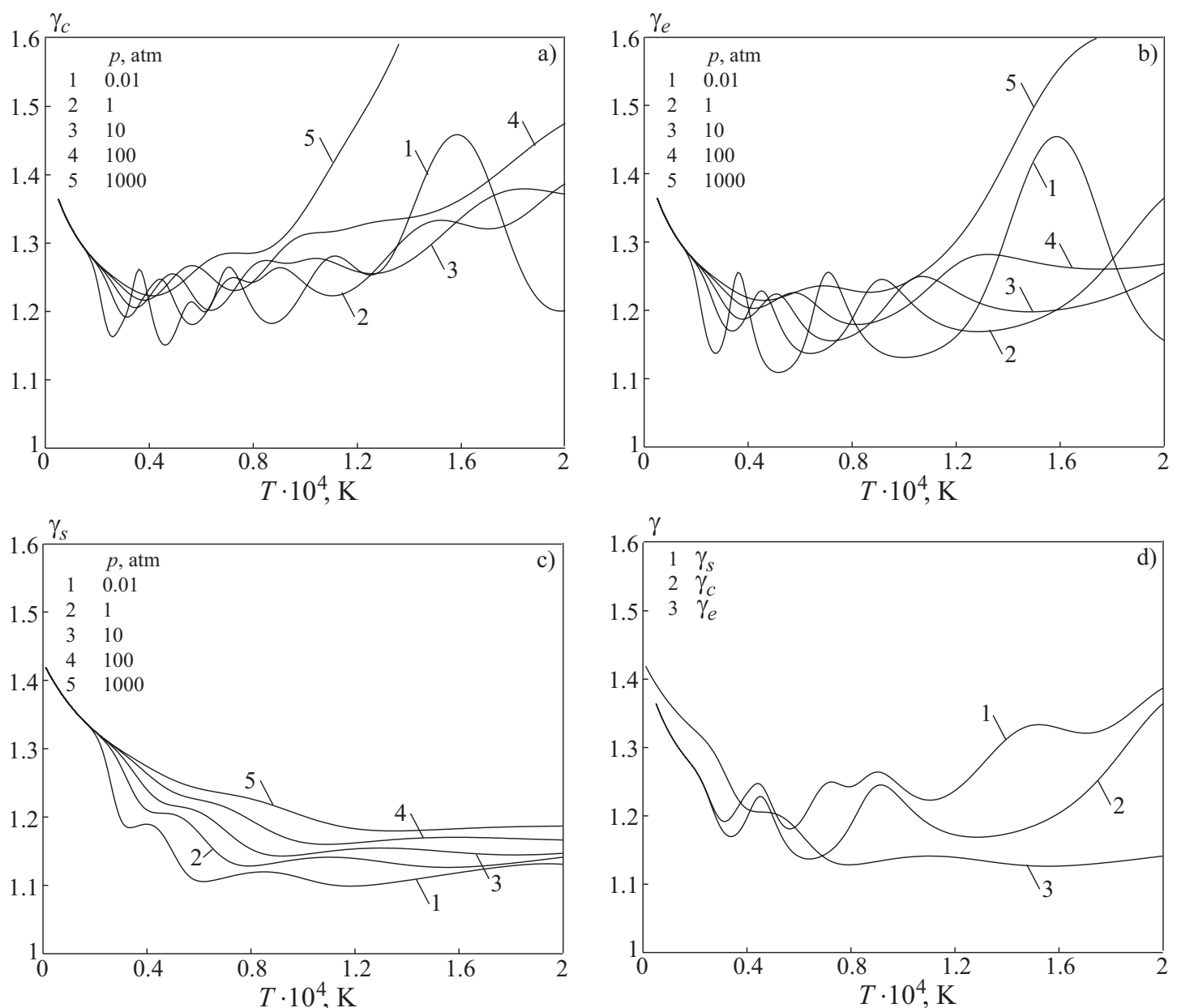

Figure 1. Dependencies of the effective ratio of specific heat capacities on temperature and pressure

\section{$5 \quad$ Model of energy supply}

In calculations of the flows of an ideal gas, simplified energy supply models are used which do not take into account processes of ionization and dissociation, as well as chemical reactions that occur in a real gas at high temperatures [10].

\subsection{Temperature distribution}

The $T$-model assumes an instantaneous energy supply and zero value of the source term in the energy conservation equation $(q=0)[26]$. The energy supply is specified by the temperature distribution at the initial time moment $t=0$. The source term describes an ellipse centered at $\left(x_{0}, y_{0}\right)$, and the dimensions of the half-axes $R_{x}$ and $R_{y}$ are determined as

$$
T(x, y)=T_{\infty}+\Delta T(x, y)
$$

where

$$
\Delta T(x, y)=\Delta T_{0} \exp \left[-\left(\frac{x-x_{0}}{k R_{x}}\right)^{2}-\left(\frac{y-y_{0}}{k R_{y}}\right)^{2}\right]
$$

Here, $T_{\infty}$ is the temperature of surrounding gas, and $\Delta T_{0}=T_{0}-T_{\infty}$ is the temperature increment at the center of energy supply region. The smaller the parameter $0.5 \leqslant k \leqslant 1$, the 
smaller the gap in gas parameters at the boundary of the energy supply zone is observed. The value of the determining parameter of the model $\Delta T_{0}$ is calculated by the formula

$$
\Delta T_{0}=\frac{Q}{\rho_{\infty} c_{v} I}
$$

where $Q$ is the energy absorbed by the medium, $\rho_{\infty}$ is the density of surrounding gas, and $c_{v}$ is the specific heat capacity at constant volume. The integral of the function describing the spatial distribution of intensity in the volume $V_{0}$ has the form

$$
I=\int_{V_{0}} \exp \left[-\left(\frac{x-x_{0}}{k R_{x}}\right)^{2}-\left(\frac{y-y_{0}}{k R_{y}}\right)^{2}\right] d V .
$$

This model simulates only a single pulse of energy supply, not taking into account its duration.

\subsection{Intensity distribution}

In $q$-model, it is possible to take into account the influence of stationary, single, pulse or periodic supply of energy of various durations and frequencies. In this case, the source term in the energy conservation equation is non-zero $(q \neq 0)[26]$.

The specific power supplied to the region in the form of an ellipse centered at the point $\left(x_{0}, y_{0}\right)$, and the dimensions of the semi-axes $R_{x}$ and $R_{y}$ are determined as

$$
q(t, x, y)=q_{0} f(t) \exp \left[-\left(\frac{x-x_{0}}{k R_{x}}\right)^{2}-\left(\frac{y-y_{0}}{k R_{y}}\right)^{2}\right],
$$

where $q_{0}$ is the specific power of the energy supply at the center, $f(t)$ is the function describing the intensity change in time, $R_{x}$ and $R_{y}$ are the characteristic lengths of the energy release region. The parameter $0.5 \leqslant k \leqslant 1$ determines the intensity values at the boundary of the energy supply region. For a single pulse of energy of duration $\tau$ function $f(t)$ has the form

$$
f(t)= \begin{cases}1 & \text { if } 0 \leqslant t<\tau \\ 0 & \text { if } \tau \leqslant t\end{cases}
$$

The determining parameter of the model is calculated by the formula

$$
q_{0}=\frac{Q}{\rho_{\infty} \tau I} .
$$

When the energy supply simulates the operation of a three-phase current plasma torch, in which the areas of energy release (electric arcs) periodically appear and move along the channel centreline, the coordinates $x_{0}=x_{0}(t)$ and $y_{0}=y_{0}(t)$ are determined by the path travelled by the arc and the speed.

In case of setting the mass energy supply, the effective energy supply is several times smaller due to a decrease in the gas density during heating [27]. Available results indicate the promise of using simplified models of energy supply for an ideal perfect gas during parametric calculations [10]. 


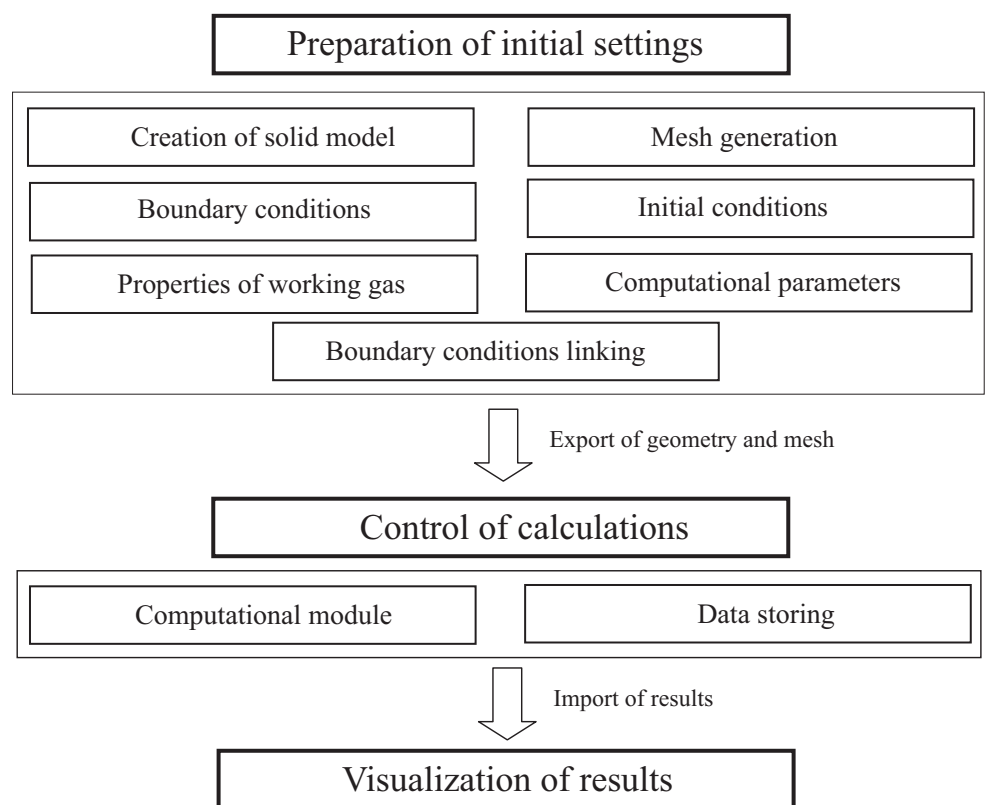

Figure 2. Functional sub-systems of the code

\section{Code structure}

The code consists of three main sub-systems, a data preparation sub-system (pre-processor), a counting process control sub-system (CFD solver), and a visualization sub-system (postprocessor). Interaction of sub-systems and data streams are shown in the Figure 2).

Functioning of various sub-systems is as follows.

1. Sub-system of data preparation and creation of a solid state model. Import solid models and meshes from commercial packages.

1.1. Setting the geometry of the computational domain. Geometry information is stored separately from the flowfield.

1.2. Meshing.

1.3. Specification of boundary conditions. Permissible types of boundary conditions and the necessary data for specifying each of these types. Boundary conditions are linked to individual mesh lines. The process is based on commercial mesh generators.

1.4. Specification of initial conditions. Initial conditions are set either analytically or using table interpolation data, or by reading from a file.

1.5. Setting parameters related to the physical formulation of the problem and the implementation of the computational algorithm.

2. Sub-system of control the process of counting. Several control options are used:

- management during the calculation is not allowed (management is done through the data preparation system, setting the number of time steps, the results are written in files);

- partial control (the possibility of observation is provided to change some parameters);

- full control (suspension of the account and change of control parameters, preservation of the current state with subsequent restart, obtaining intermediate results, outputting results at reaching a given value of a parameter).

3. Sub-system of visualization of results (graphical representation of the results in the form of contours, vector fields, graphs) and export results in a number of formats supported by common commercial packages.

From a mathematical point of view, calculating the flow quantities is a solution of the initial/boundary value problem using the finite volume method (Figure 3).

In a conventional programming language, the scheme for solving the problem is as 


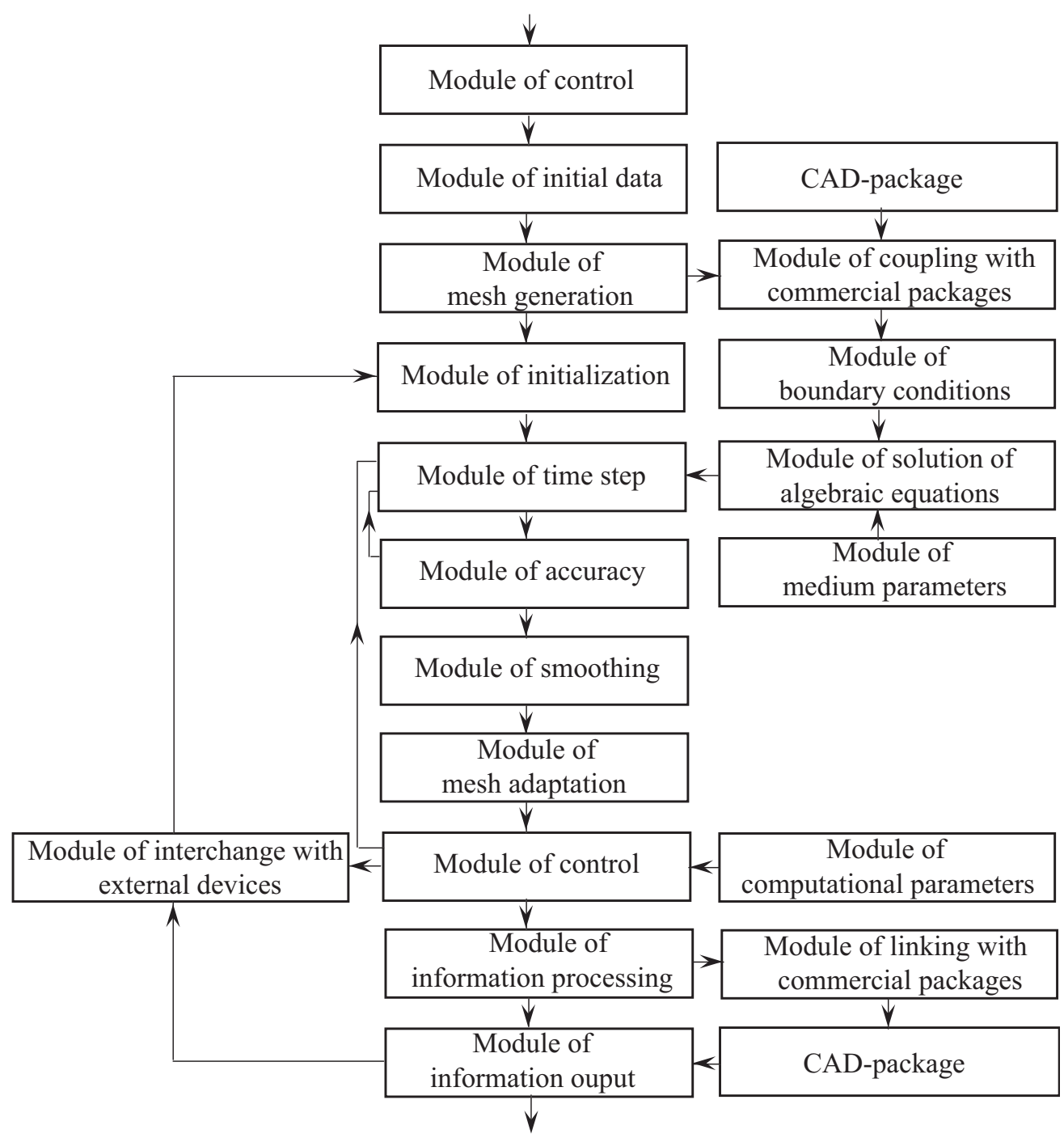

Figure 3. Structural scheme of the problem solution

follows.



Creating a solid state model and meshing, specification of boundary conditions and processing of the results are performed on the local computer using commercial data processing systems. The mesh is uploaded in a text or binary format. The pre-processor reads the mesh file and generates a file containing necessary information about the number and size of the vectors that are allocated in memory at the calculation stage. The solver reads this file, file containing the boundary and initial conditions and file containing physical parameters of the environment. The calculation is performed, and the calculated data is uploaded to a file for subsequent processing in the visualization system. The memory is allocated at the data loading stage, and iterative calculation is performed without memory reallocation. 
Powerful and flexible technology for software development is provided by object-oriented programming (OOP) tools [28-30]. Its distinguishing features are transparency and the ability to access details of the implementation of a particular method and algorithm, the mathematical clarity of the description of the new method for the developer and user, the openness and the ability to supplement the library with new procedures, and the ease of use of the developed methods.

A mathematical class is associated with each of the distinguished concepts, and a problem class is associated with a generalized statement (Figure 4).

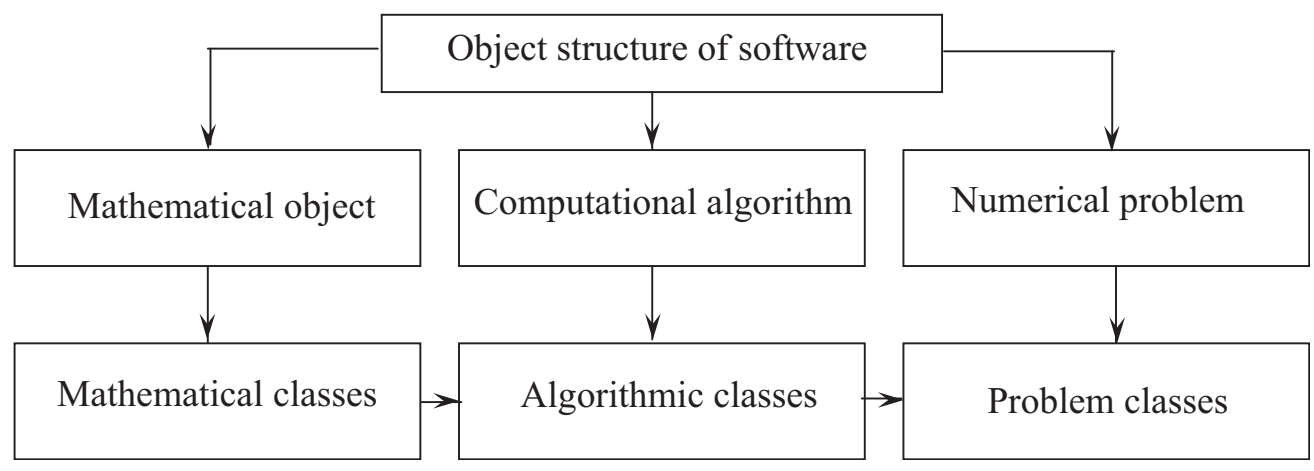

Figure 4. Object structure of package and class hierarchy

A mathematical object is an entity expressing a certain mathematical category and making up an object of computation. In the class core of the library are included vectors, matrices, and, in the more general case, the geometric primitives from which the solid model is built. Each mathematical object has a set of mathematical features that are the basis for classification constructions. However, mathematical objects do not constitute a computational problem and are only a tool for its formulation and solution. Mathematical classes express general problem-invariant concepts, and a mathematical object-oriented library is a basic tool environment for developing computing applications.

The computational algorithm refers to the methods of computational mathematics and supporting information that determines the conditions for their algorithmic use. Each algorithm is designed to solve one problem, although it can be indirectly used to solve other problems (different approaches can be used to solve numerical problems of one class). In addition to the parameters of the numerical method, the algorithmic objects contain information about the accuracy of the solution and the computational resources available (they are expressed, for example, in the form of a limit on the number of iterations and counting time).

The problem of computational mathematics, presented in a unified form (for example, the problem of solving a system of differential equations), is considered a numerical problem. The organization of problem classes provides the desired commonality of software implementation of close problem statements that differ in the types of mathematical objects. Along with solving the problem, it is important to have information about the correctness and efficiency of using the algorithm in a specific situation. Such information is associated with the problem being solved, and not with the used computing objects. Since objects of numerical problems have a longer lifetime than basic objects, computing resources are managed at this level.

Files and data required for the numerical solution of the problem in one way or another are shown in the Figure5 (it is believed that the program works in batch mode, the graphical interface is not used).

The script file (source data file) contains the information necessary to solve the problem, in particular, the names of the files with the geometry of the region, the type of boundary 


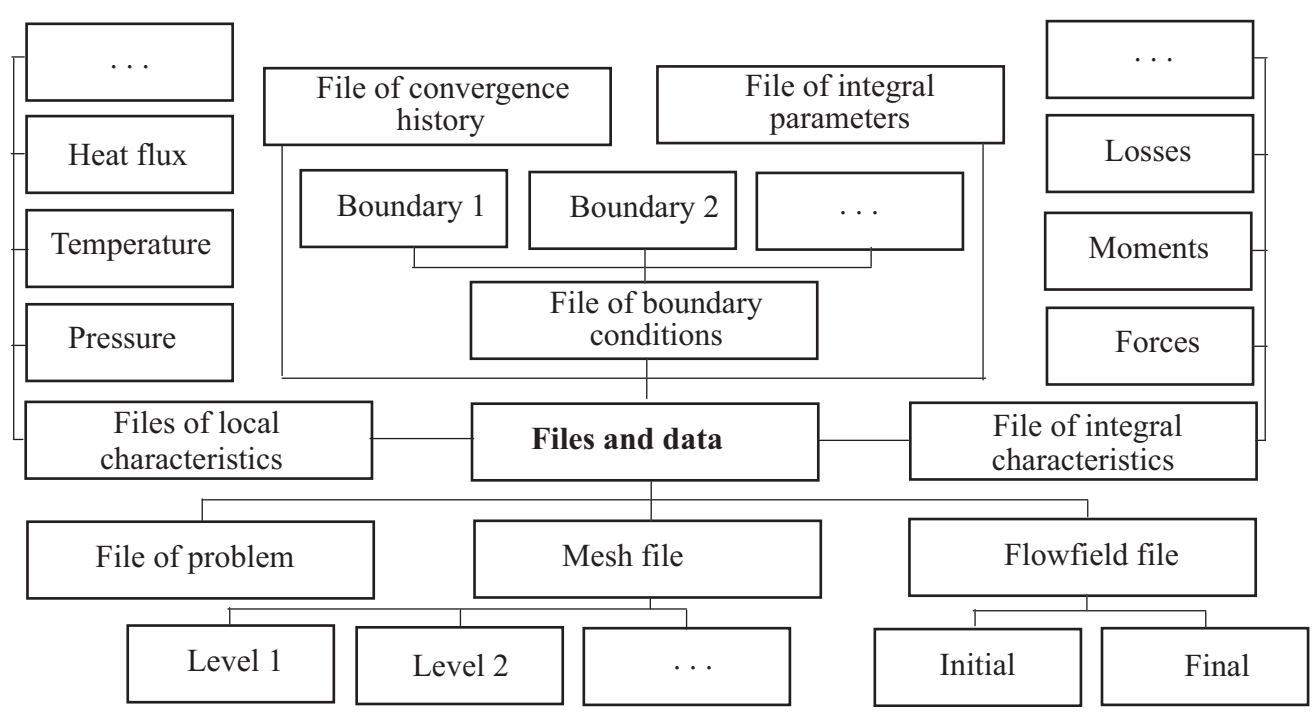

Figure 5. Files and data

conditions, the parameters of the difference scheme, the thermophysical characteristics of the liquid and other parameters. For parameters whose values are not defined in the script file, default values are used.

Binary mesh files and flow fields contain the coordinates of the mesh nodes, the initial distributions of the desired functions, as well as the shape of mesh cells, the type of boundary conditions to be set, and a number of other parameters. The mesh is stored separately from the flowfield. At the same time, a separate file is used to store each level of the unstructured mesh (level 1 corresponds to the mesh with the best resolution). The initial and final flowfields are stored either in separate files (the file of the initial flowfield is kept unchanged) or in the same file (the file of the initial flowfield is replaced by a new one).

Boundary condition files store function profiles defined at the boundaries. To check the convergence of the iterative process, a file containing the values of the residuals is required. To process the calculation results, the values of the local and integral parameters of the stream are stored in files whose names are indicated in the script file.

\section{$7 \quad$ Results and discussion}

Results of numerical simulation of one-dimensional and two-dimensional under- and overexpanded nozzle flows with a moving region of energy supply are presented.

\subsection{Nozzle geometry and energy supply}

The channel cross-sectional area varies according to the dependence $S=1+2 x^{2}$, where $-0.3 \leqslant x \leqslant 1$. The coordinate $x=0$ corresponds to the critical section of the nozzle. For one-dimensional calculations, a mesh containing 800 nodes is used. In two-dimensional calculations, a uniform mesh is used along the coordinates $x$ and $y$ containing $800 \times 400$ nodes. The number of mesh nodes is selected based on checking the convergence of numerical solutions on different meshes with a gradual increase in relevant dimension. Calculations are terminated upon reaching a final given point in time.

The energy supply model with a moving energy release region allows one to take into account the effect of the displacement of the intense energy supply zone in case of one or more plasma torch arcs. The diagram of a single energy supply region that periodically 
appears and moves in the channel is shown in the Figure 6a. The family of lines correspond to different points in time (with a constant step), and the shape of each curve displays the spatial distribution of the energy supply intensity. The energy supply diagram for three periodic moving arcs with a time-varying intensity is shown in the Figure 6b. In both cases, three full periods of energy supply are shown, where $\tau$ is the period of energy supply, and $Q_{0}$ is the maximum value of energy supply. The arc speed is $10 \mathrm{~m} / \mathrm{s}$, and the path travelled by the arc is $0.2 \mathrm{~m}$. The arc burning time is $0.025 \mathrm{~s}$.
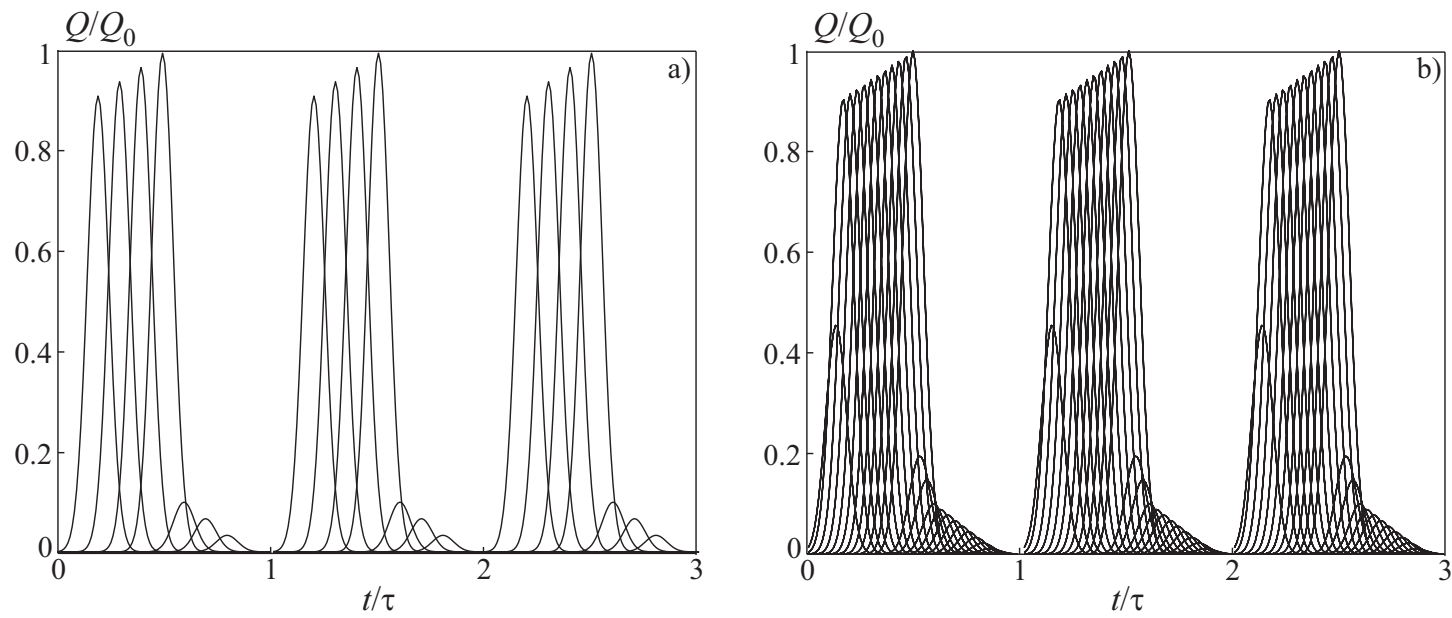

Figure 6. Distribution of the energy supply intensity over time for one arc (a) and three $\operatorname{arcs}(\mathrm{b})$

The form of the function that describes the change in intensity over time is shown in the Figure 7 for a single arc and three arcs of a plasma torch. With a sufficiently large number of pulses, the distribution of the energy release intensity over the spatial coordinate becomes almost uniform.
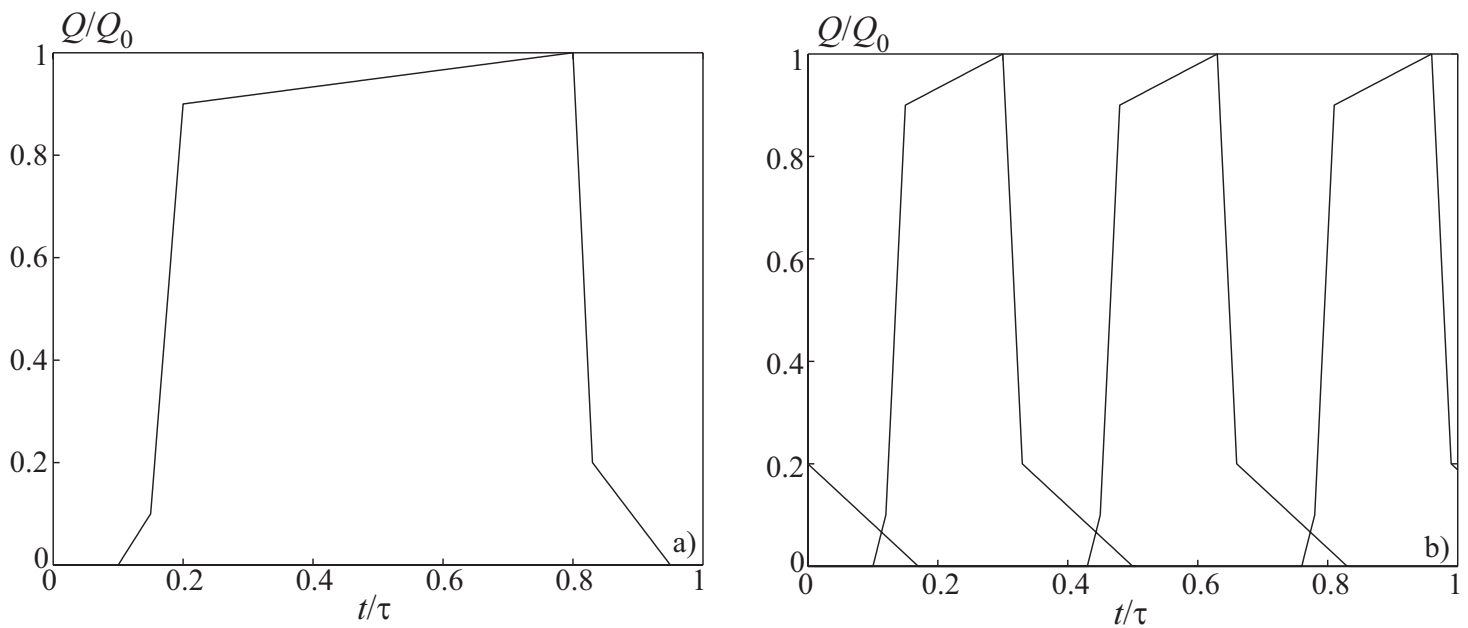

Figure 7. A single discharge pulse (a) and multiple discharge pulses (b)

The use of pulse or periodic energy sources makes it possible to realize sufficiently high peak power supply. The structure of the shock wave and the thermal wake behind the energy supply region substantially depend on the pulse repetition rate. With a certain frequency of such sources and by ensuring energy equivalence, it is possible to realize flows with properties close to those that are formed under conditions of stationary energy supply, when the thermal trace becomes continuous. The condition of energy equivalence is determined by the equality of the energy supply parameter in stationary and non-stationary cases. 


\subsection{Test cases}

The unsteady flows in a channel with a variable cross-sectional area in one-dimensional and two-dimensional formulations without an energy supply are considered, as well as a stationary one-dimensional flow with energy supply in a narrow zone (reaction front), which has an exact solution describing the flow quantities in zones before and behind power supply.

The pressure field in a two-dimensional flow in a nozzle without energy supply, obtained by the pseudo time-marching method, is shown in the Figure 8 . Such flow regimes in the nozzle are considered when a nozzle shock wave is formed inside. The nozzle shock is visiblein the region of concentration of the pressure level lines.

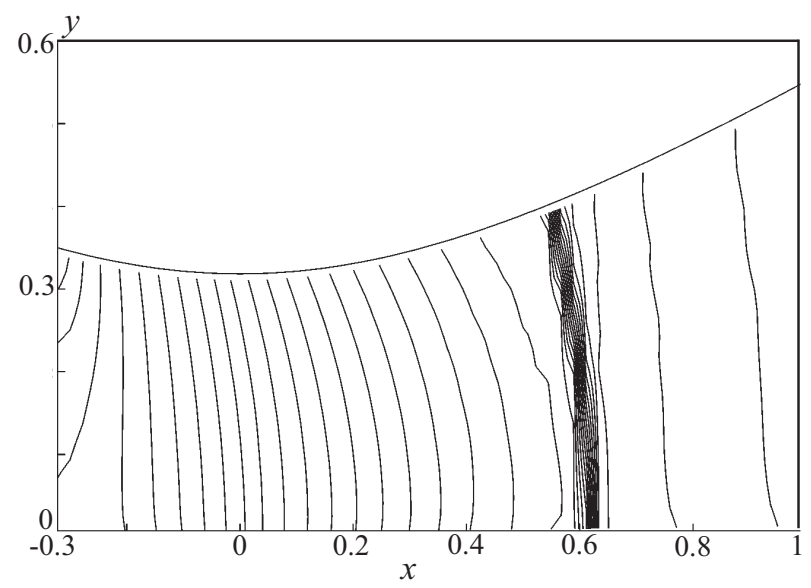

Figure 8. Contours of pressure in the nozzle flow without energy release

A comparison of the exact solution of the stationary quasi one-dimensional problem (solid line) and the limiting numerical solution of the unsteady two-dimensional problem (the symbols $\circ$ and $*$ ) is shown in the Figure 9 . Satisfactory agreement of solutions is ensured on a relatively coarse mesh, and the intensity and position of the shock wave corresponds to the exact solution. The difference scheme spreads the nozzle shock wave over 1-2 mesh cells. The solution is monotonous (there are no non-physical oscillations).

Another test case corresponds to the flow in the channel, in the output section of which the boundary conditions are set corresponding to the supersonic flow (characteristic boundary conditions). The energy supply zone is reproduced in one cell. The pressure and temperature distributions behind the front of the power supply are shown in the Figure 10 depending on the Mach number in front of the power supply zone. For each Mach number, there are two solutions, and there is a threshold of energy supply intensity (critical energy supply). When the energy supply intensity exceeds a critical value, there is no stationary solution of the problem. For example, for the Mach number $\mathrm{M}_{1}=3$, the critical energy supply is $q=Q /\left(c_{p} T_{1}\right)=1.4815$. Subscripts 1 and 2 refer to flow quantities before and behind the energy supply zone.

The distributions of the flow quantities during supercritical energy supply are shown in the Figure 11 at a fixed time, with $\mathrm{M}_{1}=3$. The dimensionless energy supply parameter is assigned the value $q=2$.

\subsection{One-dimensional flows}

The nozzle operating conditions are considered when the pressure of the over-expanded flow is restored through the nozzle shock wave. Unsteady energy supply, carried out in the section 


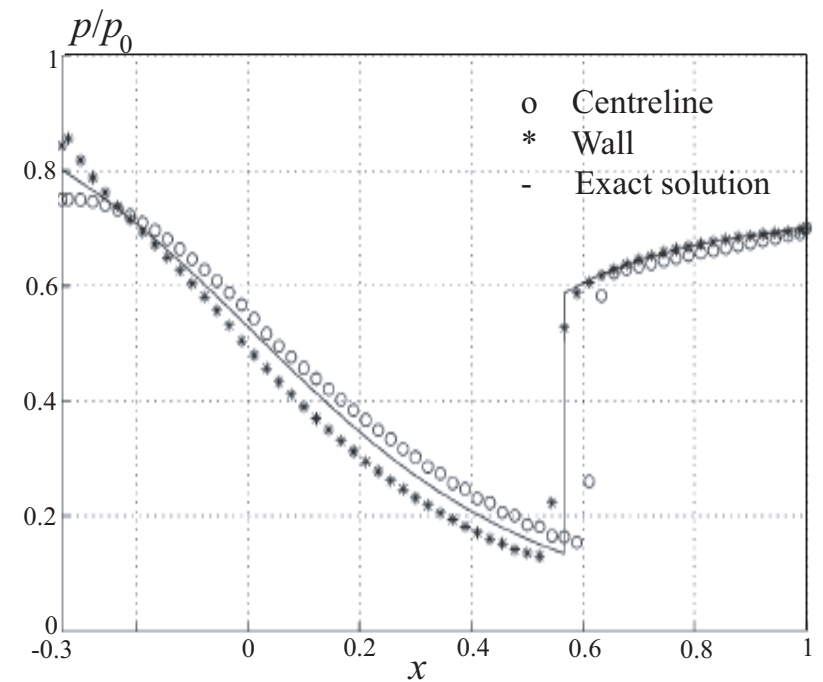

Figure 9. Comparison of exact solution (solid line) with results of numerical calculations (symbols 0 and $*)$. Symbols o correspond to the flow quantities along the centreline, and symbols $*$ correspond to the flow quantities near the nozzle wall
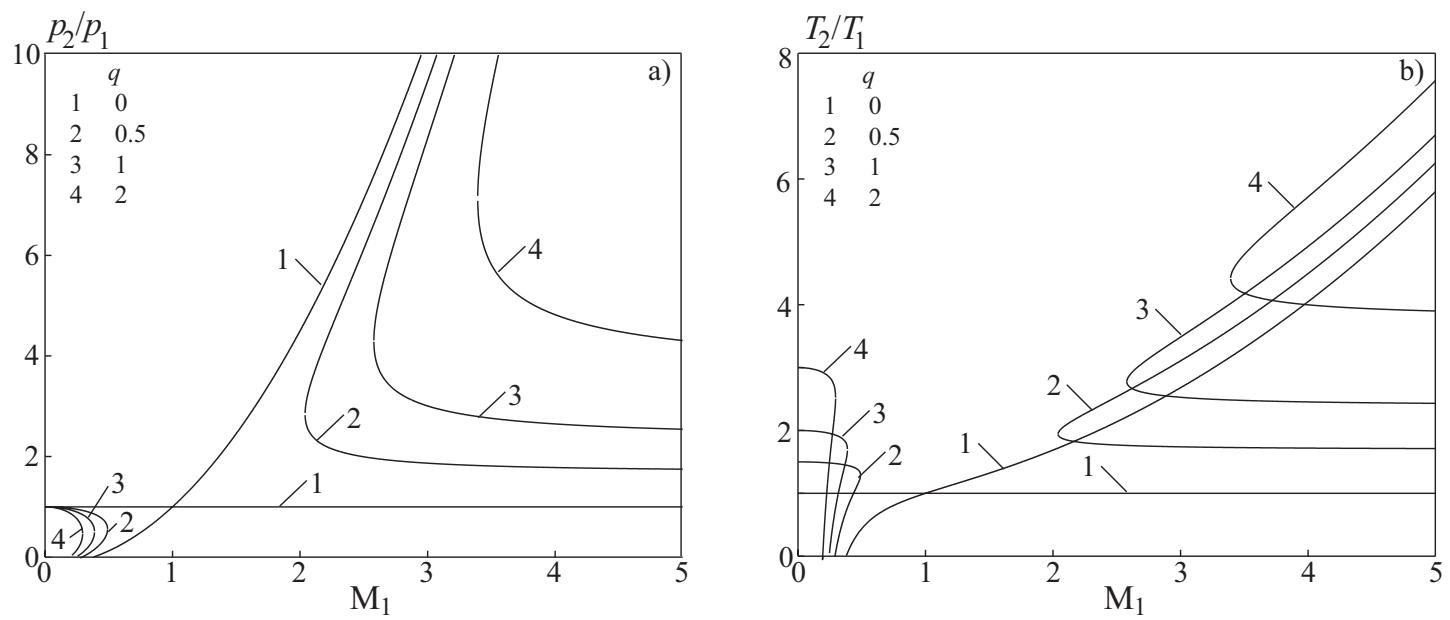

Figure 10. Pressure distributions (a) and temperature distributions (b) before and behind the energy supply region for $q=0(1), 0.5(2) ; 1$ (3); 2 (4)

of the subsonic path of the nozzle, causes the development of shock waves accompanied not only by a change in the flow quantities, but also by the movement of shock wave structures and pressure fluctuations. Unsteady energy supply leads to a significant restructuring of the nozzle flow, an increase in temperature, and a change in gas pressure near the energy supply region. After the end of the pulse, the high-temperature zone is transferred along the centreline.

The pressure and temperature distributions at different points in time are shown in the Figure 12 and Figure 13. The total pressure and the total temperature in the inlet section are fixed at $8.5 \cdot 10^{5} \mathrm{~Pa}$ and $300 \mathrm{~K}$, and the static pressure in the outlet section of the nozzle is set to $6 \cdot 10^{5} \mathrm{~Pa}$. The solid lines show the pressure and temperature distributions corresponding to the stationary solution to the problem without energy supply, and the points show the pressure and temperature distributions in the unsteady case. The distributions of flow quantities given in the Figure 12a and Figure 13a correspond to the beginning of the arc ignition process. For simplicity, the position of the arc, the intensity of which has a Gaussian 


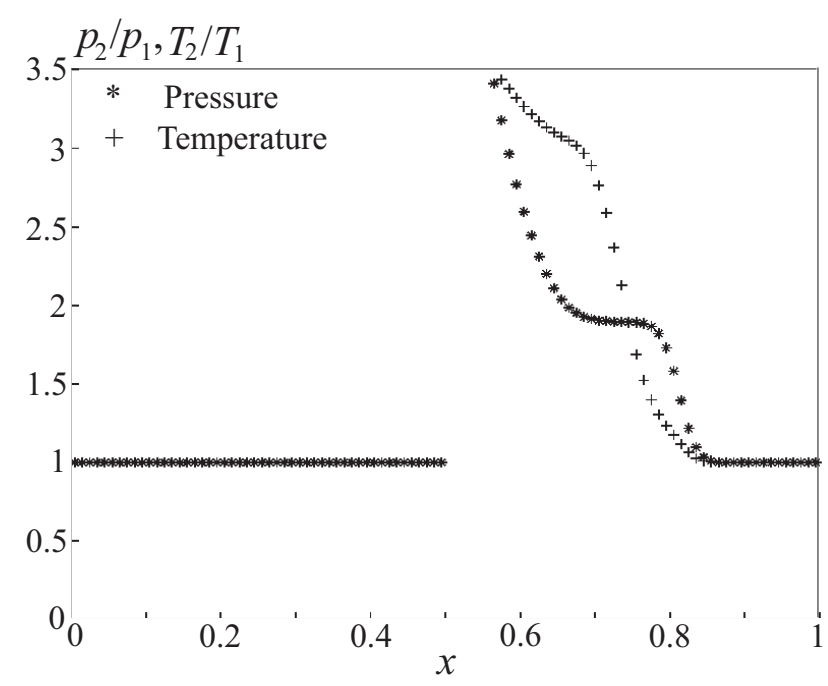

Figure 11. Pressure distributions (symbols $*$ ) and temperature distributions (symbols + ) at time $t=1.546$

distribution in space with a sufficiently high localization, is shown by a vertical line for the initial period of the energy supply cycle. The energy supply causes the appearance of high gradients of flow quantities, localized in a narrow region. The process development is shown in the Figure 12bd and Figure 13b-d. With a change in the intensity of energy supply, which corresponds to the ignition and extinction of an arc, the intensity of discontinuities changes, and regions with sharp gradients of the flow quantities move along the nozzle.

The effect of unsteady energy supply is shown in the Figure 14, where $q=Q_{0} /\left(G_{0} H_{0}\right)$ is the dimensionless parameter that determines the energy supply intensity, and $G_{0}$ and $H_{0}$ are the mass flow rate and total enthalpy without energy supply. If there is a periodic energy supply in the subsonic part of the nozzle, the gas flow through the critical section also becomes a periodic function. The integral value of the flow rate decreases compared to its value in a flow without energy supply, and this difference increases with an increase in the energy supply intensity.

For alternating current plasmatrons, the energy supply option with several simultaneously moving arcs is of interest. Figure 15 and Figure 16 show the effect of heat release on the pressure and temperature distributions for three arcs (these results correspond to one energy supply cycle). The arcs move one after the other along the nozzle centreline. The vertical lines correspond to the positions of the arc at the corresponding time moments. For simplicity, the spatial distribution of the energy supply intensity is not shown in the figures.

Due to the fact that the arcs move periodically, the distributions of flow quantities in any sections are also periodic functions (Figure 17). An increase in the number of arcs leads to a more uniform temperature distribution along the nozzle centreline.

\subsection{Two-dimensional flows}

In the two-dimensional case, energy is supplied in the subsonic part of the nozzle. The energy supply region moves along the nozzle centreline, and the energy supply intensity cyclically changes in time. The case of one burning arc is considered.

The pressure and temperature distributions at different times of the energy supply cycle are shown in the Figure 18 and Figure 19. At moderate intensities of energy supply, its effect on the pressure field is manifested to a lesser extent than on other flow quantities. The origin 

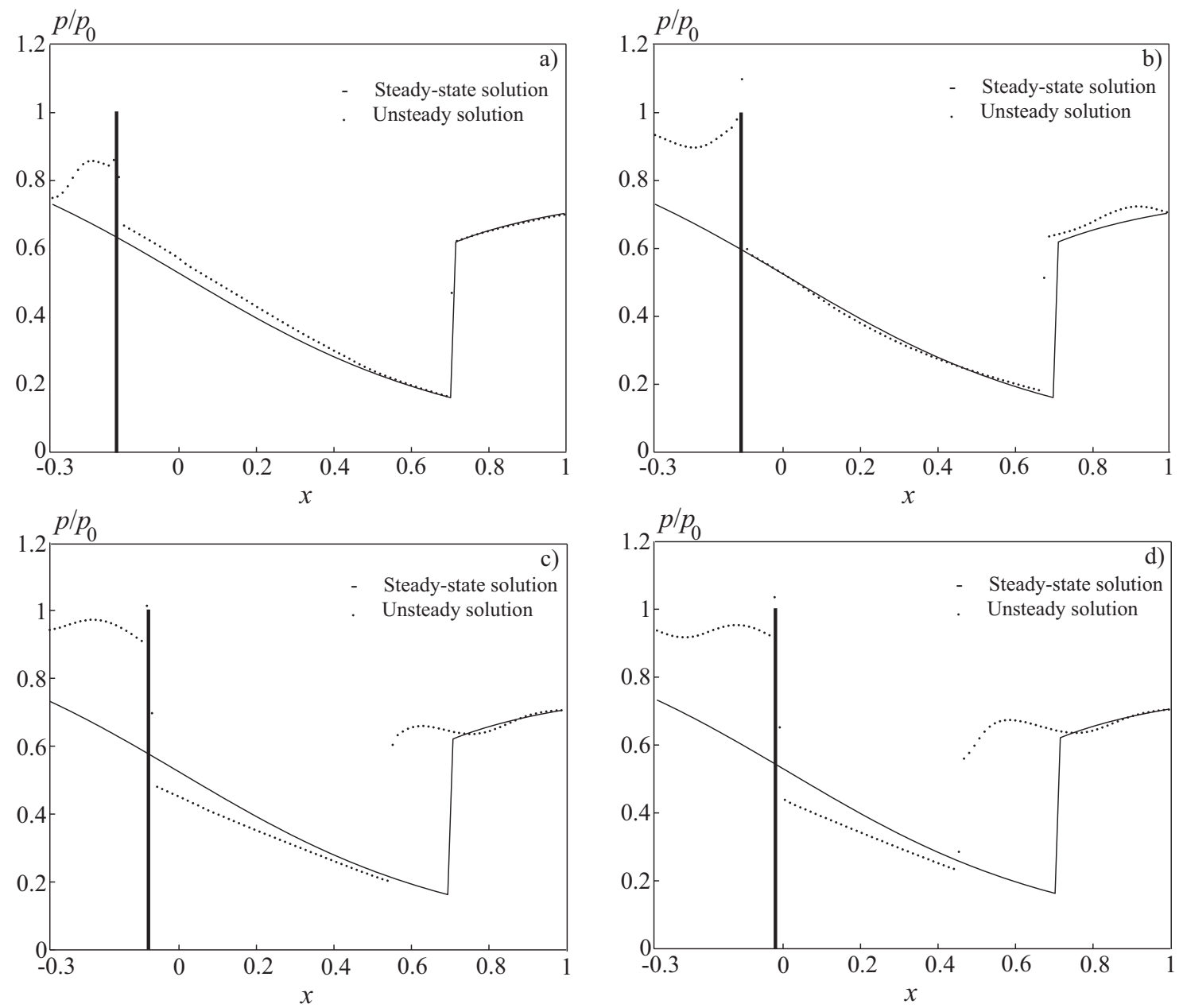

Figure 12. Pressure distributions at time $t / \tau=1.156$ (a), 1.396 (b), 1.516 (c), 1.724 (d) for one arc

of the high-temperature region shows the Figure 18a and Figure 19a, which correspond to the moment the arc begins to burn. The high-temperature region is transferred by the flow. The length of this region increases in time, and temperature gradients increase as well (Figure 18b and Figure 19b). Behind the shock wave, a trace of the previous heat spot is visible. Further development of the high-temperature region is observed in Figure $18 \mathrm{c}$ and Figure 19c. After the arc attenuation, a convective drift of the heat spot occurs, and the temperature field becomes the same as during a flow without energy supply (Figure 18d and Figure 19d). The configuration of the perturbed region and the amplitude values of the flow quantities (temperature, pressure, Mach number) depend on the size of the energy release region and the maximum value of the energy input.

The effect of the energy supply on the distribution of flow quantities is shown in Figure 20 and Figure 21 for a fixed nozzle section. The solid lines correspond to the critical section of the nozzle, and the dash-dotted lines correspond to the outlet section of the nozzle. The influence of the energy supply on the temperature field is much more significant than that on the pressure field. The pressure distributions inside and outside the energy supply region are similar. The results obtained allow concluding that there is a significant non-uniform flow in the outlet section of the nozzle. Both the temperature field and the pressure field have two areas of sharp changes in parameters. In addition to the nozzle shock wave, sharp gradients of flow quantities are observed in a narrow region of intense energy release. 

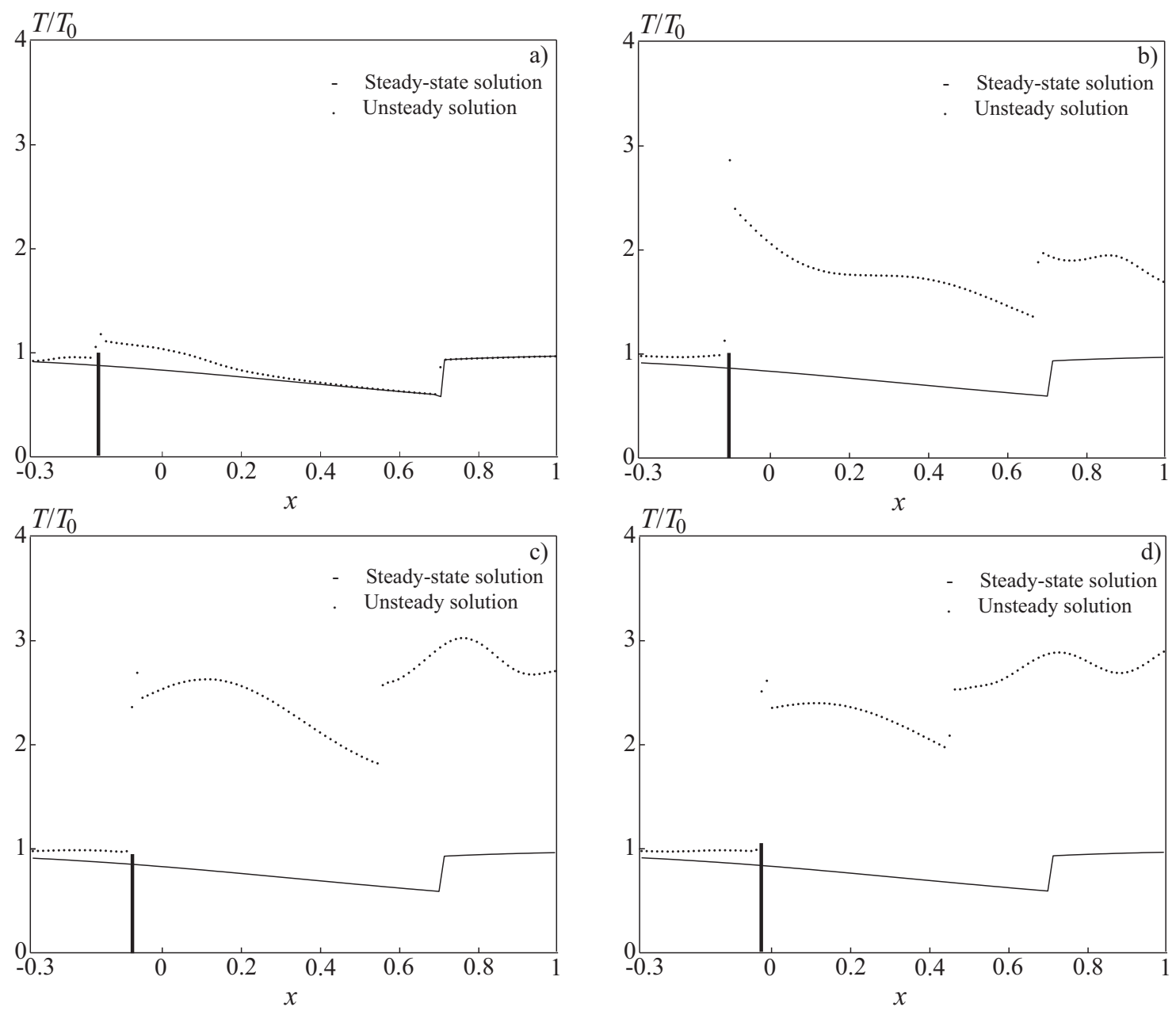

Figure 13. Temperature distributions at time $t / \tau=1.156$ (a), 1.396 (b), 1.516 (c), 1.724 (d) for three arcs
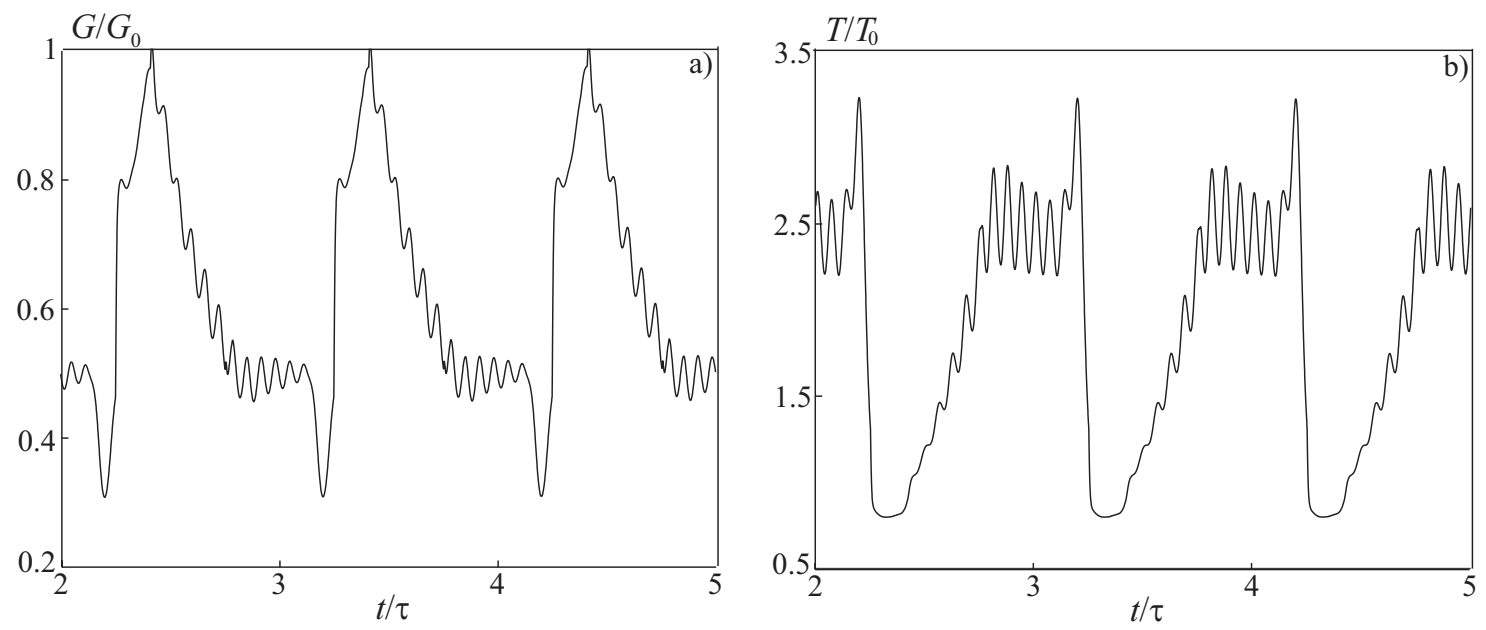

Figure 14. Mass flow rate through critical section of the nozzle (a) and temperature distribution in the critical section (b) for one arc

\subsection{Flows of real gas}

The nozzle cross sectional area changes as $S(x)=1+2 x^{2}$, where $-1 \leqslant x \leqslant 2$. The total temperature at the inlet section of the nozzle is fixed at $5000 \mathrm{~K}$. At the nozzle exit, a pressure is set corresponding to the case of subsonic outflow. The calculations are carried out using 

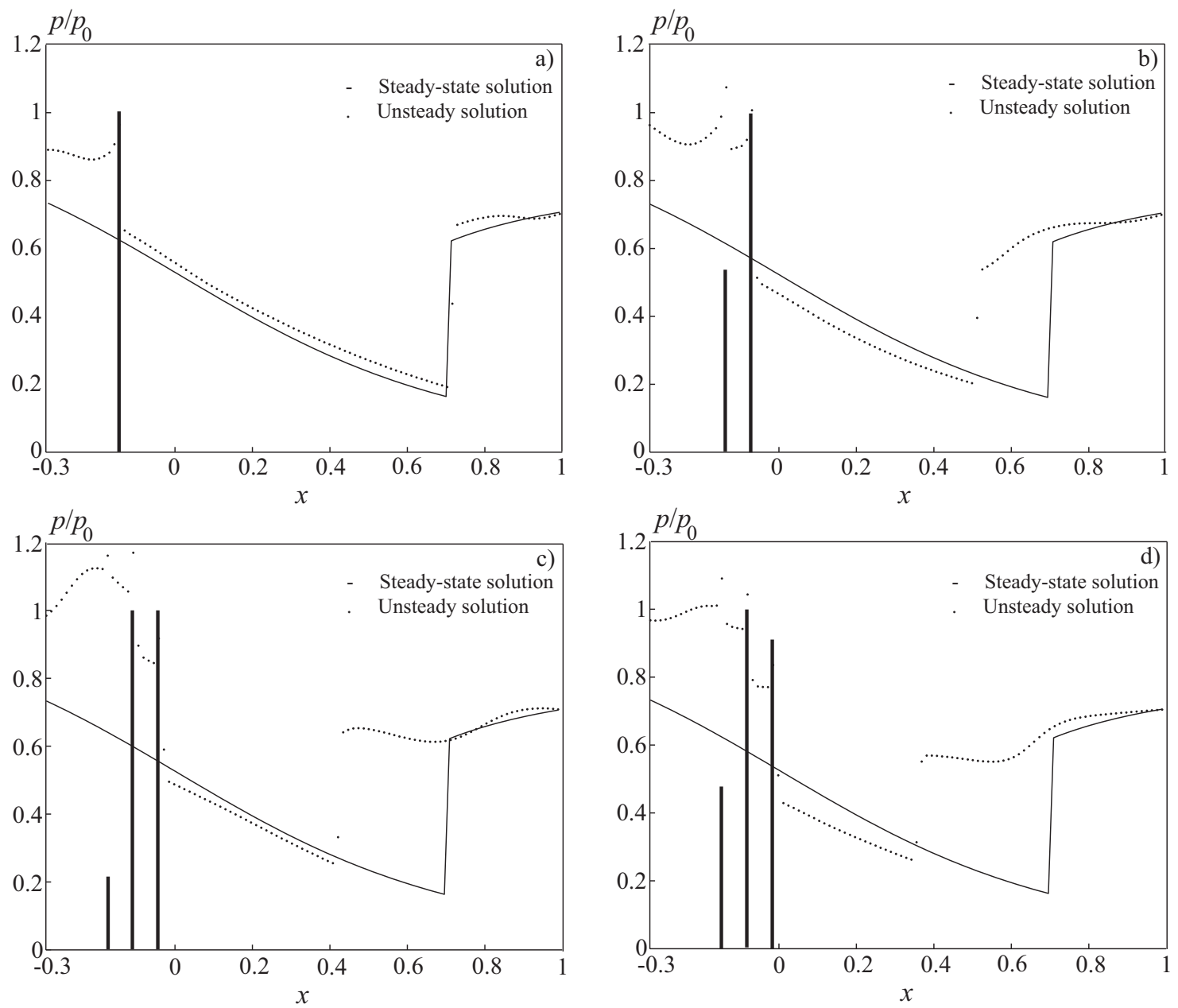

Figure 15. Pressure distributions at time $t / \tau=1.224$ (a), 1.544 (b), 1.668 (c), 1.768 (d) for three arcs

various methods for calculating the effective adiabatic exponent $\left(\gamma_{c}, \gamma_{e}, \gamma_{*}\right)$. The results are compared with those based on ideal gas model for two values of the adiabatic index $\left(\gamma_{1}=1.4\right.$ and $\gamma_{2}=1.2$ ). The influence of various methods for determining the effective adiabatic index on the distribution of flow quantities is investigated.

Comparative distributions of pressure, temperature and Mach number for various methods for determining the effective adiabatic exponent are shown in the Figure 22 for a flow without an internal shock wave. The solid and dash-dotted lines correspond to the adiabatic exponents $\gamma_{1}=1.4$ and $\gamma_{2}=1.2$, and the symbols $\circ, *$ and + correspond to the adiabatic exponents $\gamma_{c}, \gamma_{e}$ and $\gamma_{s}$. In the calculations corresponding to ideal and real gas flows, equal values of temperature and pressure are set at the nozzle inlet. Moreover, the total enthalpy of real gas is much higher than for an ideal gas.

Comparative distributions of pressure, temperature, and Mach number are shown in the Figure 23 during the flow through the nozzle with the formation of an internal shock wave (the notation is the same as in the Figure 22). The change in various effective adiabatic exponents along the nozzle centreline is shown in the Figure 24 (the solid line corresponds to $\gamma_{c}$, the dashed line corresponds to $\gamma_{s}$, the dash-dotted line corresponds to $\gamma_{e}$ ). The pressure distribution in the nozzle flow with a subsonic output depends both on the magnitude of the adiabatic index and method for determining its effective value. The distribution of the Mach number is more sensitive to the method of determining the adiabatic exponent. Using a real gas model leads to significant deviations in the temperature distribution. Due to 

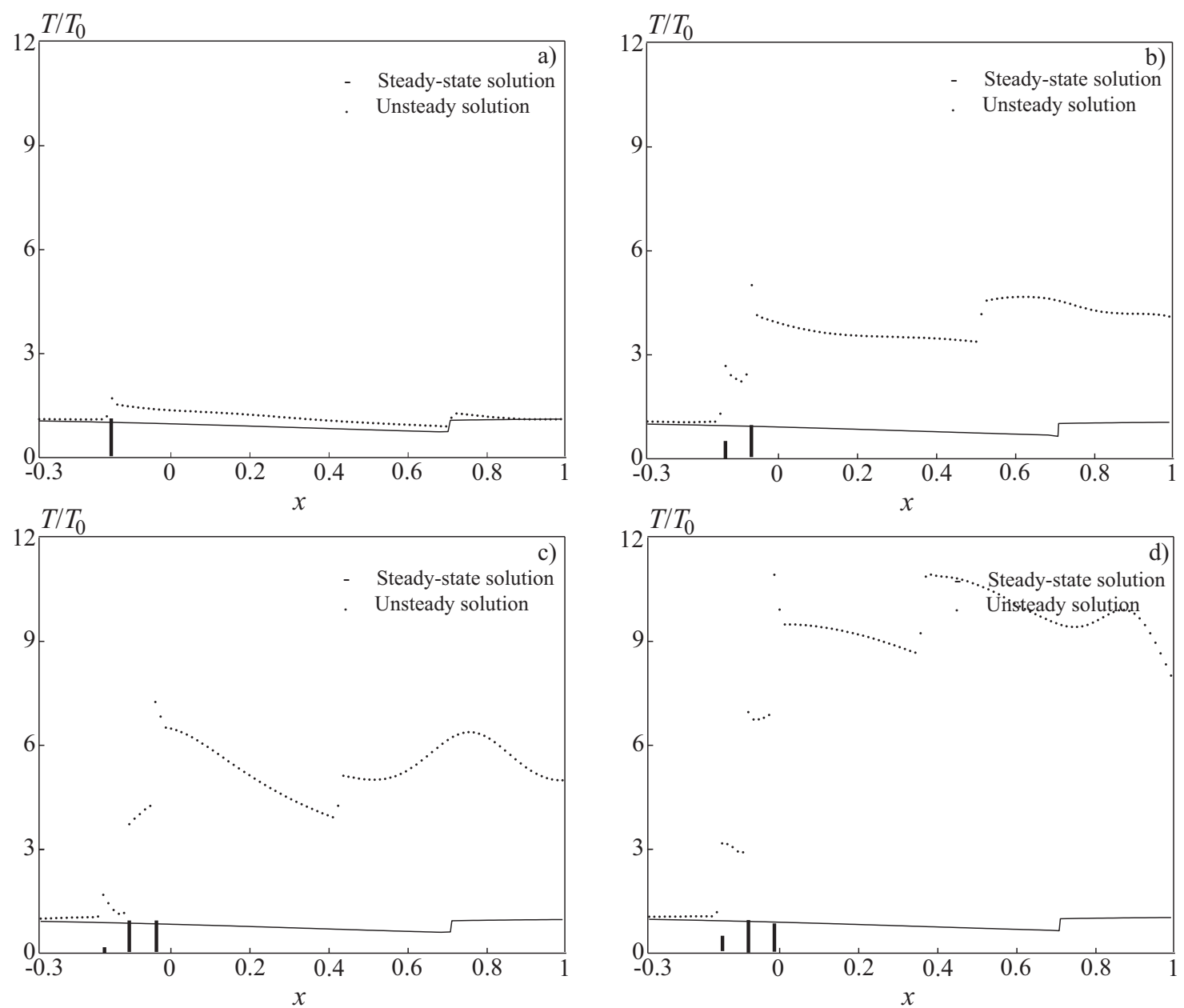

Figure 16. Temperature distributions at time $t / \tau=1.224$ (a), 1.544 (b), 1.668 (c), 1.768 (d) for three arcs


Figure 17. Mass flow rate through the critical section of nozzle (a) and temperature distribution in the critical section of nozzle (b) for three arcs

the significantly different dissociation energies for oxygen and nitrogen, oxygen begins to dissociate earlier than nitrogen. Behind the shock wave, in accordance with an increase in temperature, the degree of dissociation also rises sharply.

Another option for comparing ideal and real gas flows, corresponding to the case of an under-expanded flow in the nozzle, is shown in the Figure 25a. The same total pressure and 

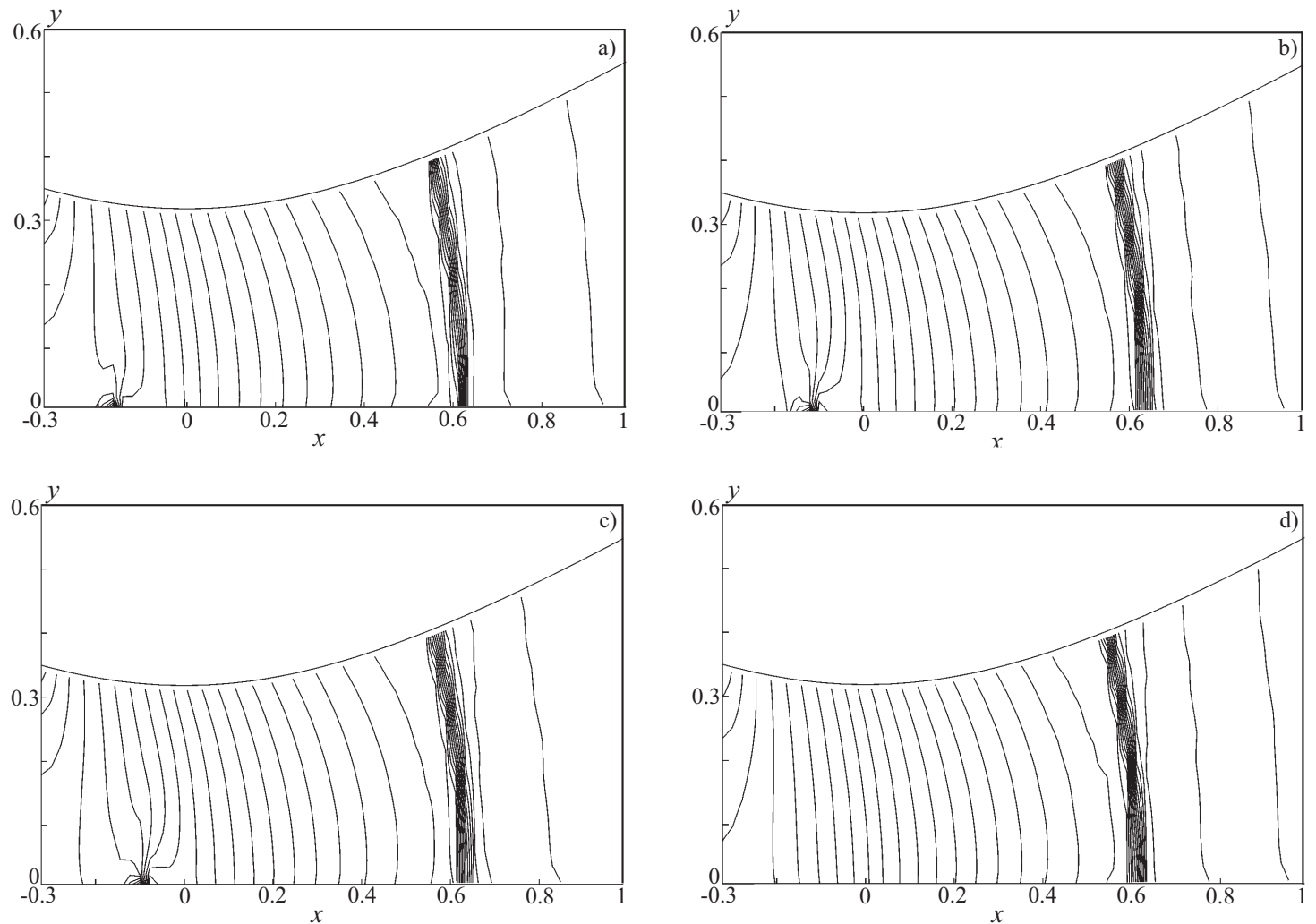

Figure 18. Contours of pressure at time $t / \tau=2.154$ (a), 2.410 (b), 2.531 (c), 2.977 (d)
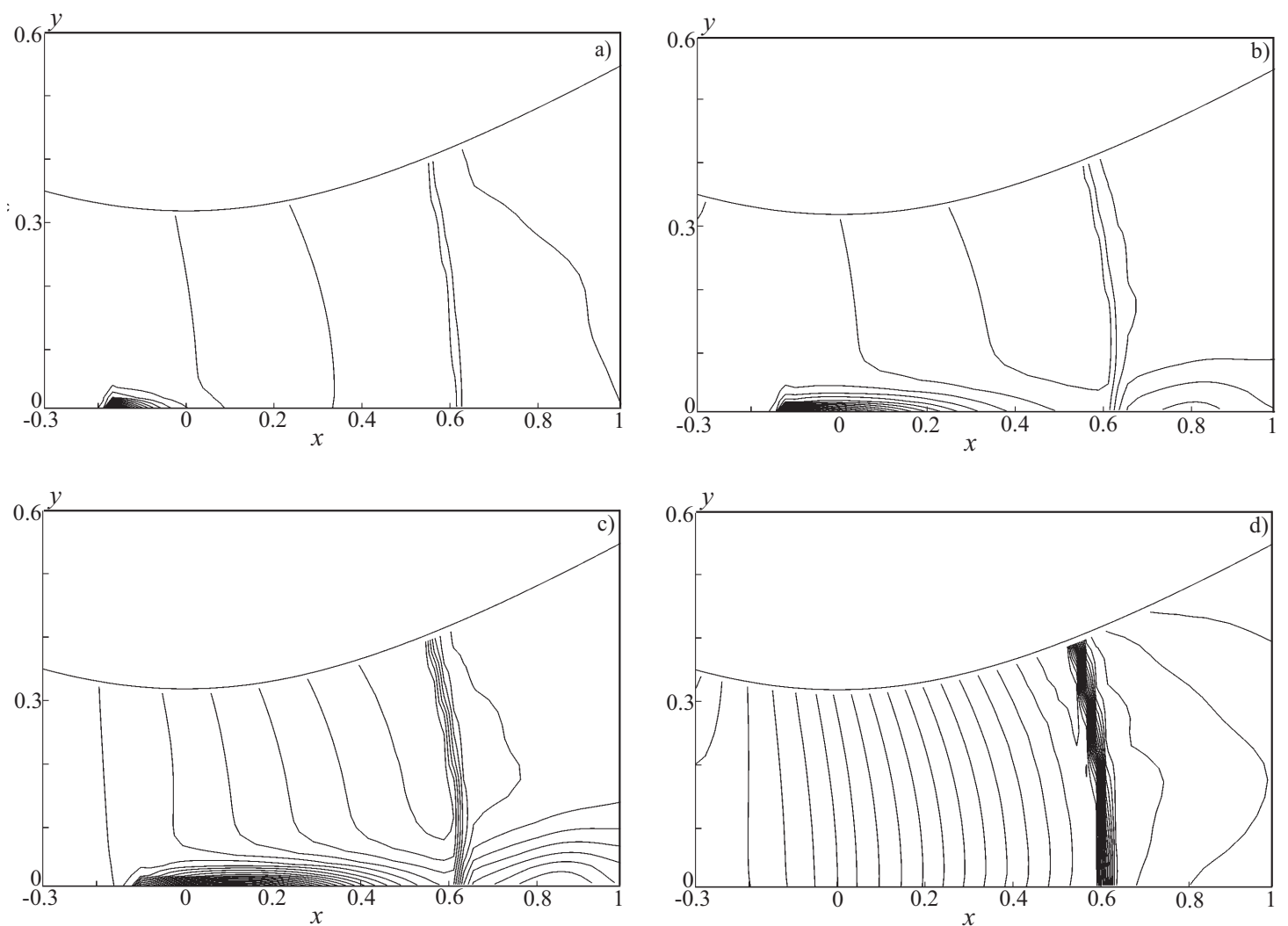

Figure 19. Contours of temperature at time $t / \tau=2.154$ (a), 2.410 (b), 2.531 (c), 2.977 (d) 

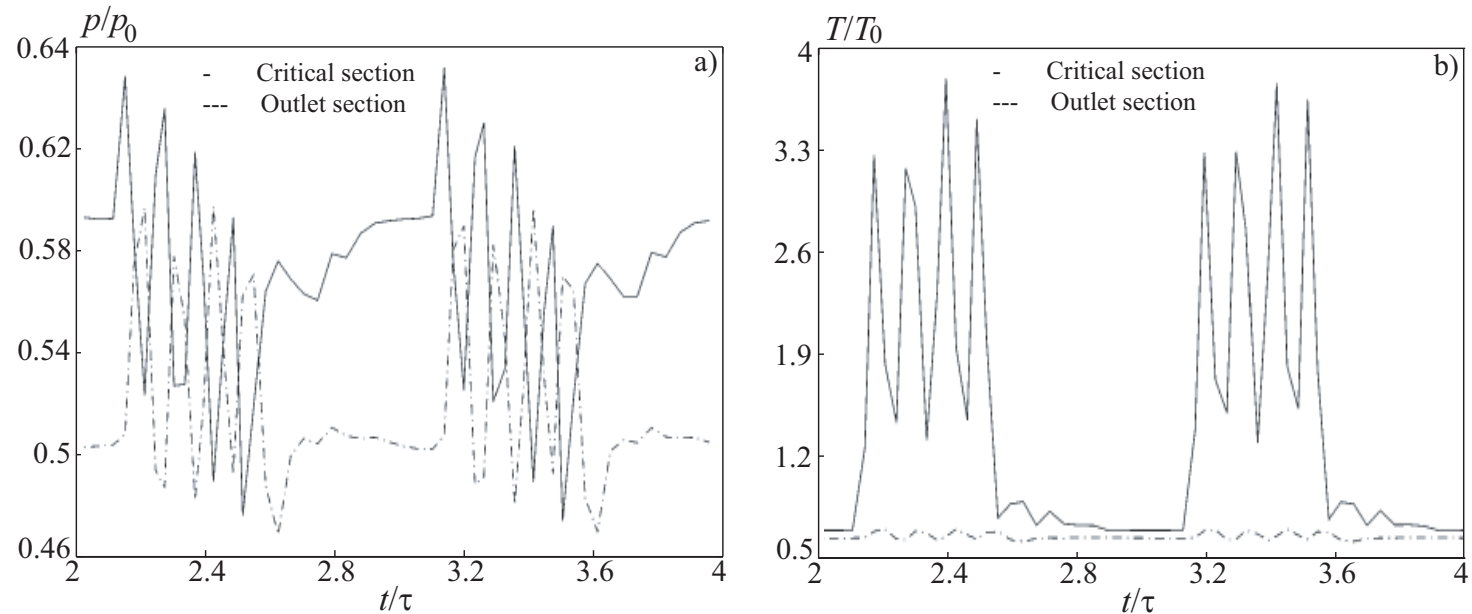

Figure 20. Pressure (a) and temperature (b) distributions along nozzle centreline $(x=0)$
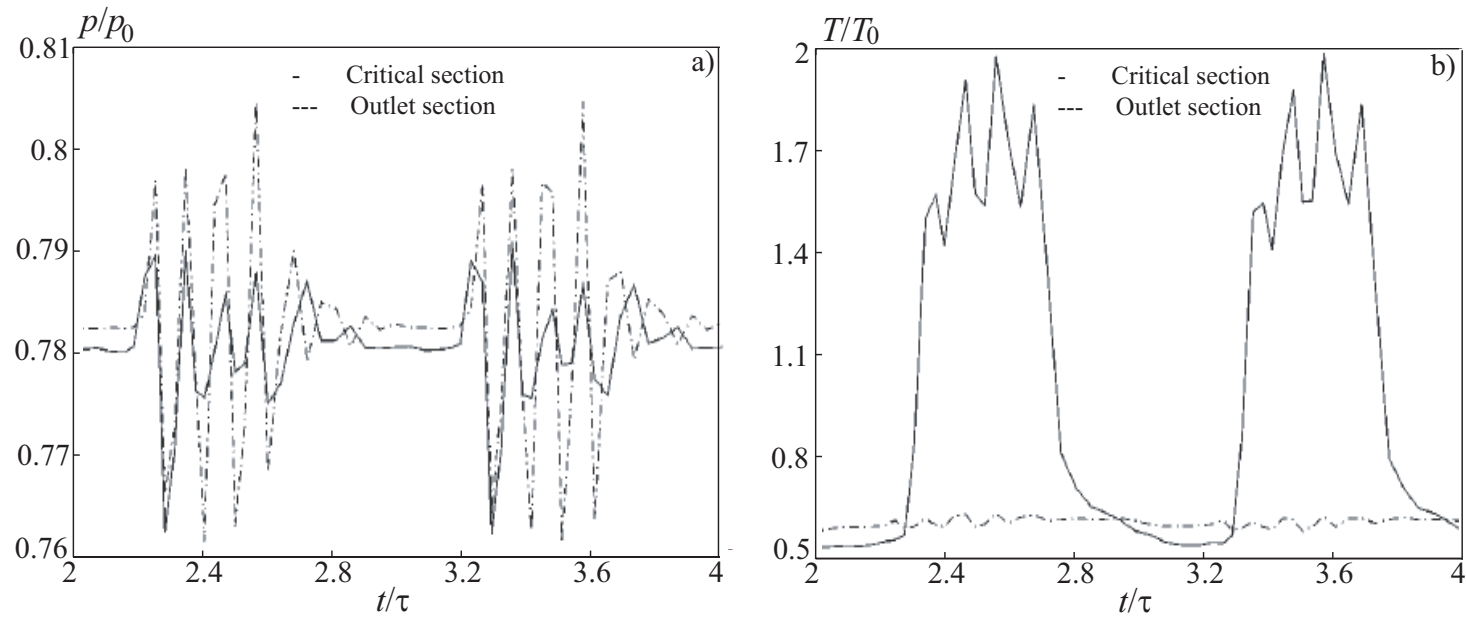

Figure 21. Pressure (a) and temperature (b) distributions near nozzle wall $(x=0.98)$

total enthalpies are taken as conditions at the nozzle inlet. The temperature distribution in real gas is much lower than in ideal gas. This is because real gas absorbs significant energy for the dissociation of molecules. For another calculation option, corresponding to the case of an over-expanded flow in the nozzle, the temperature is assumed to be $10000 \mathrm{~K}$ (Figure 25b). For such conditions, ionization processes in the input section become noticeable.

A real gas model allows taking into account dissociation processes. The proportion of dissociated oxygen and nitrogen molecules depends on the current temperature and pressure. It should be noted that, due to the significantly different dissociation energies for oxygen $(5.1 \mathrm{eV})$ and nitrogen $(9.8 \mathrm{eV})$, oxygen begins to dissociate earlier than nitrogen. By the time nitrogen dissociation begins, oxygen dissociation is almost complete (Figure 26a). The ionization process is just starting, and the dissociation of oxygen completely finishes to the start of ionization. The change in the degree of dissociation of oxygen and nitrogen molecules in the case of over-expanded outflow from the nozzle is shown in the Figure 26b. Behind the shock wave, in accordance with an increase in temperature, the degree of dissociation also rises sharply. 

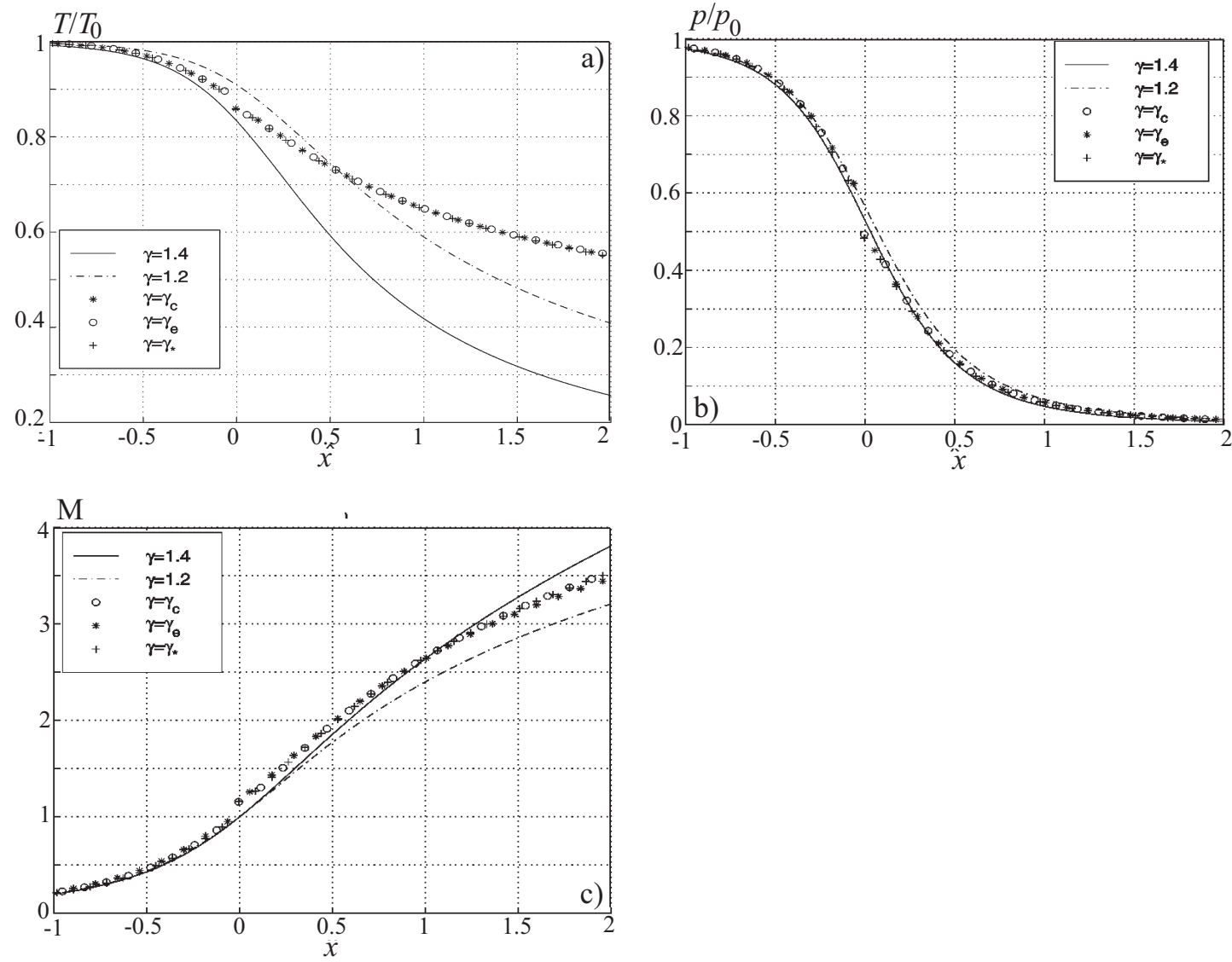

Figure 22. Temperature (a), pressure (b) and Mach number (c) distributions for various effective specific heat ratios for under-expanded nozzle flow

\section{Conclusion}

Numerical studies of gas flows in technical devices in which processes associated with unsteady supply of energy are carried out. To calculate one-dimensional and two-dimensional nozzle flows with unsteady energy supply in the subsonic part of the nozzle, the finite volume method was applied in the framework of ideal and real gas models.

The operating conditions of the nozzle with significant over-expansion are considered, when the pressure of the over-expanded flow is restored through the nozzle shock wave. The dependencies of the flow quantities on the intensity of energy supply and its effect on the position and intensity of the nozzle shock wave are reported. The supply of thermal energy to the flow leads to a significant increase in temperature and a change in gas pressure near the energy supply region. After the end of the pulse, the zone of high temperature is transferred along the nozzle centreline.

Unsteady energy supply, carried out in the subsonic part of the nozzle, causes the development of intense shock wave processes, accompanied not only by a change in the flow quantities in the nozzle, but also by the movement of shock waves and pressure oscillations. The result of energy release in the flow is an increase in temperature and pressure, but a significant decrease in the Mach number downstream. In the presence of a periodic energy supply, the mass flow rate through the critical section becomes a periodic function. The integral value of the flow rate decreases compared to its value in a flow without an energy supply, and with an increase in the energy supply intensity, this difference increases. At moderate intensities of energy supply, its effect on the pressure field is manifested to a lesser extent than on other flow quantities. The configuration of the perturbed region and the 

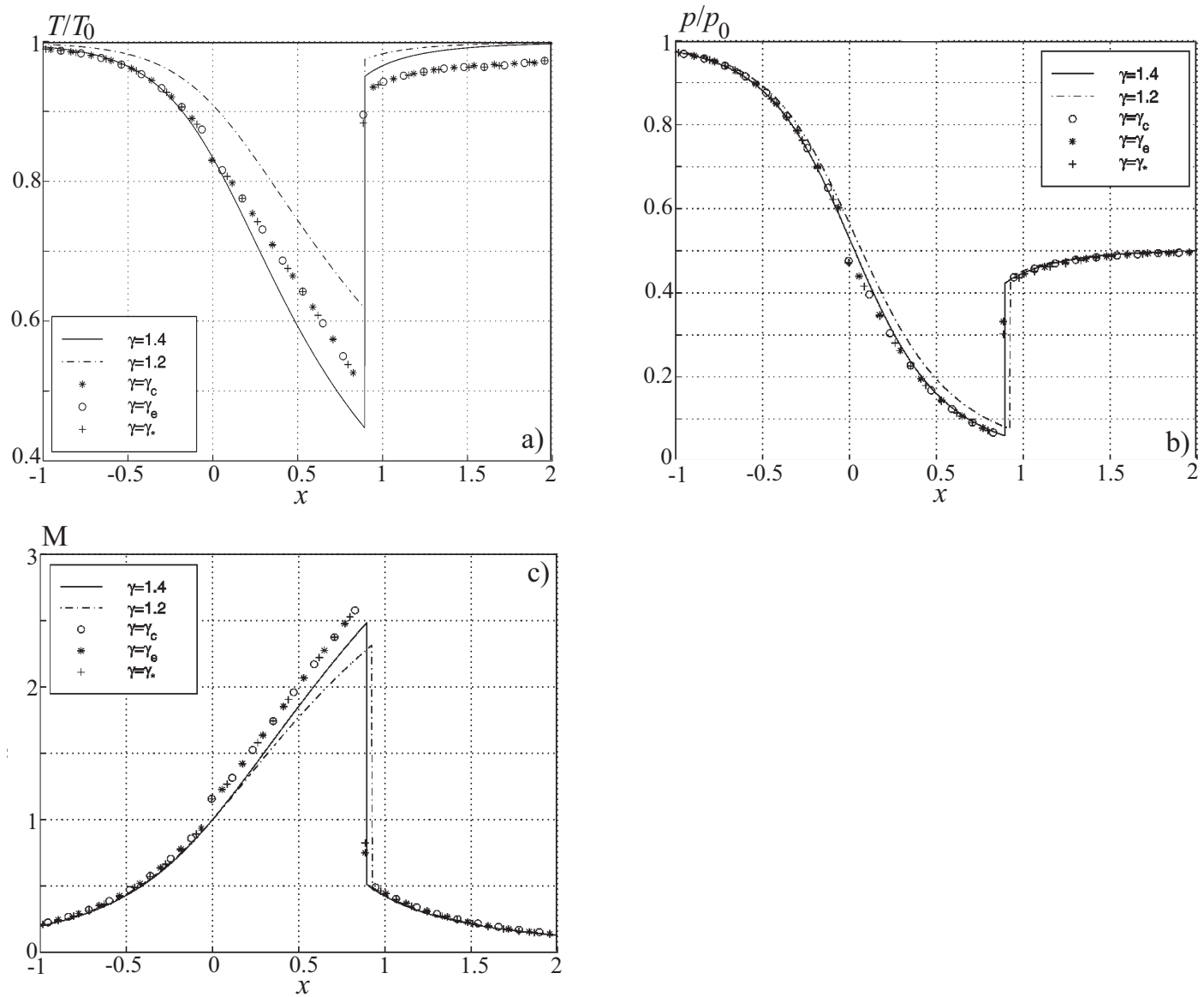

Figure 23. Temperature (a), pressure (b) and Mach number (c) distributions for various effective specific heat ratios for the over-expanded nozzle flow

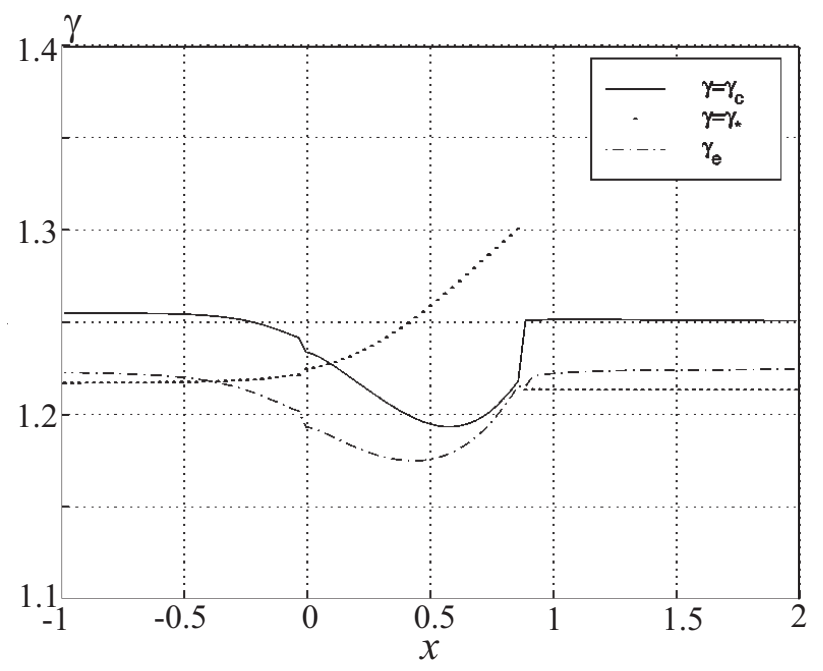

Figure 24. Distributions of effective specific heat ratios along the nozzle centreline

amplitudes of the flow quantities (pressure, temperature, Mach number) depend on the size of the energy release region and the maximum energy input. 

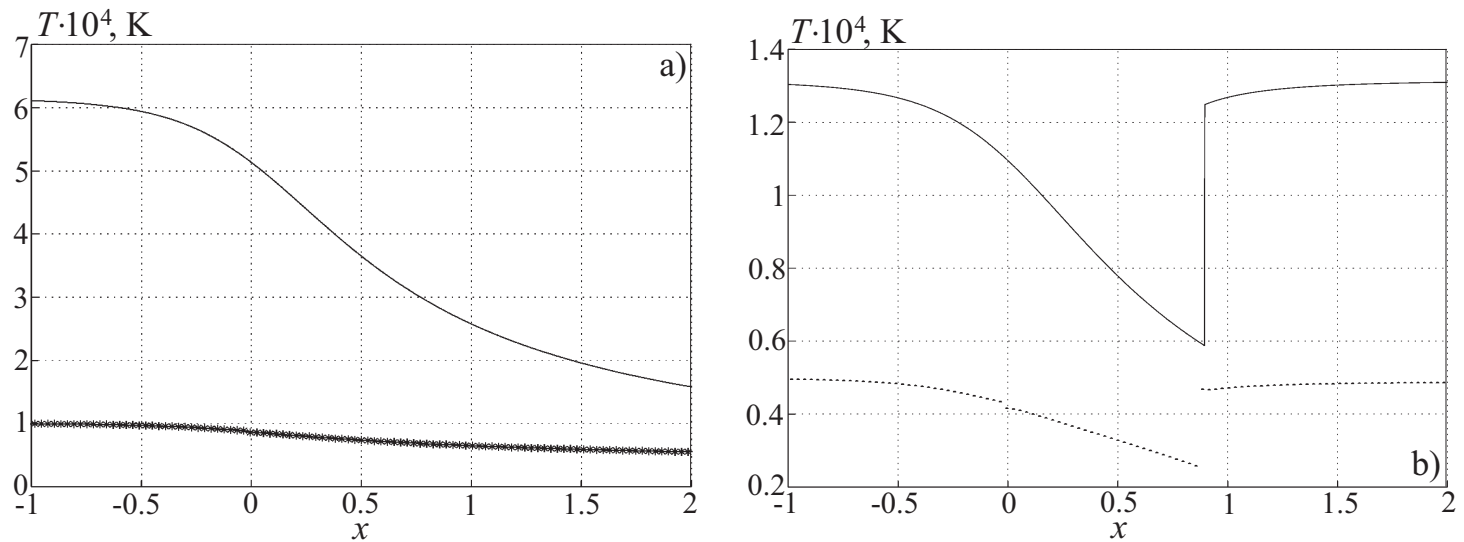

Figure 25. Temperature distributions of ideal gas and real gas for under-expanded (a) and over-expanded (b) nozzle flows
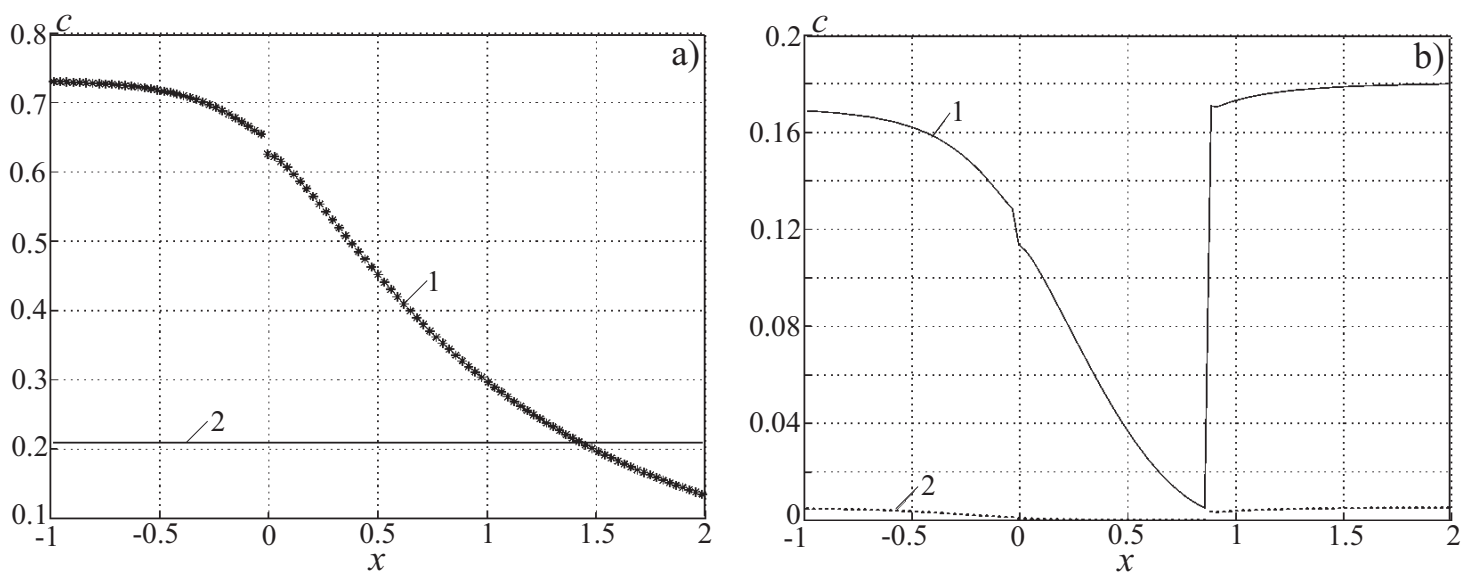

Figure 26. Distributions of molar concentrations of dissociated oxygen molecules (line 1) and nitrogen (line 2) for under-expanded (a) and over-expanded (b) flows

\section{Acknowledgements}

The study was financially supported by the Russian Science Foundation (project No. 19-7110019).

\section{References}

[1] J.D. Anderson, Hypersonic and high temperature gas dynamics. AIAA, 2006.

[2] V.P. Zamuraev, A.P. Kalinina, Gas-dynamic effects of periodic energy input into a divergent channel, Technical Physics, 55(1) (2010) 40-43.

[3] V.A. Zabaikin, I.E. Naumov, P.K. Tretyakov, Change in the regimes of flow and combustion in a channel under external energy action, Journal of Engineering Physics and Thermophysics 85(6) (2012) 1331-1338.

[4] A. Chikitkin, M. Petrov, R. Dushkov, E. Shifrin, Aerodynamic design of a Laval nozzle for real gas using hodograph method, Aerospace 5 (2018) 96-112.

[5] A.F. Latypov, Numerical simulation of the flow in a variable-section channel with pulsed-periodic energy supply, Applied Mechanics and Technical Physics 50(1) (2009) $3-11$. 
[6] M.T.C. Fang, Q. Zhuang, M.Y. Shen, The computation of axisymmetric, supersonic nozzle arc using adaptive grids, IEEE Transactions on Plasma Science 22(3) (1994) $228-234$.

[7] J.D. Yan, M.T.C. Fang, C. Jones, Electrical and aerodynamic behaviour of arcs under shock conditions, IEEE Transactions on Plasma Science 25(5) (1997) 840-845.

[8] S.T. Surzhikov, Mathematical models of subsonic Laval nozzles of laser-plasma accelerators, High Temperature 33(3) (1995) 435-448.

[9] I.V. Yegorov, D.V. Ivanov, Simulation of the flow with non-equilibrium chemical reactions in a channel of various cross section, Mathematical Modelling 9(11) (1997), $85-100$.

[10] A.A. Zheltovodov, E.A. Pimonov, Numerical simulation of an energy deposition zone in quiescent air and in a supersonic flow under the conditions of interaction with a normal shock, Technical Physics 83(2) (2013) 170-184.

[11] K. Kubo, Y. Miyazato, K. Matsuo, Study of choked flows through a convergent nozzle, Journal of Thermal Science 19(3) (2010) 193-197.

[12] N.M. Bakhtian, M.J. Aftosmis, Analysis of inviscid simulations for the study of supersonic retropropulsion, AIAA Paper 2011-3194.

[13] M. Pelanti, L. Quartapelle, L. Vigevano, A review of entropy fixes as applied to Roe's linearization, Aerospace and Aeronautics Department of Politecnico di Milano (2001).

[14] M.H. Carpenter, High-order entropy stable formulations for computational fluid dynamics, AIAA Paper 2013-2868.

[15] A. Harten, J.M. Hyman, Self-adjusting grid methods for one-dimensional hyperbolic conservation laws, Journal of Computational Physics 50 (1983) 235-269.

[16] V.N. Emelyanov, A.V. Pustovalov, K.N. Volkov, Supersonic jet and nozzle flows in uniform-flow and free-vortex aerodynamic windows of gas lasers, Acta Astronautica 163 (2019) 232-243.

[17] K.N. Volkov, Multigrid and preconditioning techniques in CFD applications, in: Z. Driss, B. Necib, H.-C. Zhang, CFD Techniques and Thermo-Mechanics Applications, Springer, 2018, pp. 83-149.

[18] V.A. Levin, V.G. Gromov, N.E. Afonina, Numerical analysis of the effect of local energy supply on the aerodynamic drag and heat transfer of a spherically blunted body in a supersonic air flow, Applied Mechanics and Technical Physics 41(5) (2000) 915-922.

[19] A.N. Kraiko, V.E. Makarov, Explicit analytic formulas defining the equilibrium composition and thermodynamic functions of air for temperatures from 200 to 20000K, High Temperature 34(2) (1996) 202-213.

[20] P. Glaister, An approximate linearised Riemann solvers for the Euler equations for real gases, Journal of Computational Physics 74(2) (1988) 382-408.

[21] A.Yu. Smenov, A modified Courant-Isaacson-Rees method for gas dynamics with an arbitrary equation of state, Computational Mathematics and Mathematics Physics 37(11) (1997) 1334-1340.

[22] P. Colella, P.M. Glaz, Efficient solution algorithms for the Riemann problem for real gases, Journal of Computational Physics 59(2) (1985) 264-289.

[23] M. Vinocur, Y. Liu, Equilibrium gas flow computations: an analysis of numerical formulations of conservation laws, AIAA Paper 88-0127. 
[24] B. Grossman, R.W. Walters, Analysis of flux-split algorithms for Euler's equations with real gases, AIAA Journal 27(5) (1989) 524-531.

[25] M.-S.Liou, B. van Leer, Splitting of inviscid fluxes for real gases, Journal of Computational Physics 87(1) (1990) 1-24.

[26] H. Yan, R. Adelgren, M. Boguszko, G. Elliott, D. Knight, Laser energy deposition in quiescent air, AIAA Journal 41(10) (2003) 1988-1995.

[27] A.N. Kucherov, Some problems of gas flows with definite distributed heat sources, TsAGI Scientific Notes 40(4) (2009) 3-14.

[28] A.P.Peskin, G.R. Hardin, An object-oriented approach to general purpose fluid dynamics software, Computers and Chemical Engineering 20(8) (1996) 1043-1058.

[29] P. Luksch, Parallel and distributed implementation of large industrial applications, Future Generation Computer Systems 16 (2000) 649-663.

[30] O. Munthe, H.P.Langtangen, Finite element and object-oriented implementation techniques in computational fluid dynamics, Computer Methods in Applied Mechanics and Engineering 190(8-10) (2000) 865-888. 